\title{
Mob Family Proteins: Regulatory Partners in Hippo and Hippo-Like Intracellular Signaling Pathways
}

\author{
Juan Carlos Duhart ${ }^{\star \dagger}$ and Laurel A. Raftery* \\ School of Life Sciences, University of Nevada, Las Vegas, Las Vegas, NV, United States
}

\section{OPEN ACCESS}

Edited by:

Cathie Pfleger,

Icahn School of Medicine at Mount

Sinai, United States

Reviewed by:

Madhuri Kango-Singh,

University of Dayton, United States

Terrence J. Piva,

RMIT University, Australia

Carole Poon,

Peter MacCallum Cancer Centre,

Australia

Lanfen Chen,

Xiamen University, China

*Correspondence:

Juan Carlos Duhart

juan.duhart@jefferson.edu

Laurel A. Raftery

laurel.raftery@un/v.edu

${ }^{\dagger}$ Present address:

Juan Carlos Duhart,

Department of Neuroscience,

Thomas Jefferson University,

Philadelphia, PA, United States

Specialty section:

This article was submitted to Cellular Biochemistry,

a section of the journa Frontiers in Cell and Developmental Biology

Received: 05 October 2019 Accepted: 28 February 2020

Published: 19 March 2020

Citation:

Duhart JC and Raftery LA (2020) Mob Family Proteins: Regulatory Partners in Hippo and Hippo-Like Intracellular Signaling Pathways.

Front. Cell Dev. Biol. 8:161.

doi: 10.3389/fcell.2020.00161
Studies in yeast first delineated the function of Mob proteins in kinase pathways that regulate cell division and shape; in multicellular eukaryotes Mobs regulate tissue growth and morphogenesis. In animals, Mobs are adaptors in Hippo signaling, an intracellular signal-transduction pathway that restricts growth, impacting the development and homeostasis of animal organs. Central to Hippo signaling are the Nuclear Dbf2-Related (NDR) kinases, Warts and LATS1 and LATS2, in flies and mammals, respectively. A second Hippo-like signaling pathway has been uncovered in animals, which regulates cell and tissue morphogenesis. Central to this emergent pathway are the NDR kinases, Tricornered, STK38, and STK38L. In Hippo signaling, NDR kinase activation is controlled by three activating interactions with a conserved set of proteins. This review focuses on one co-activator family, the highly conserved, non-catalytic Mps $\underline{1}$ binder-related (Mob) proteins. In this context, Mobs are allosteric activators of NDR kinases and adaptors that contribute to assembly of multiprotein NDR kinase activation complexes. In multicellular eukaryotes, the Mob family has expanded relative to model unicellular yeasts; accumulating evidence points to Mob functional diversification. A striking example comes from the most sequence-divergent class of Mobs, which are components of the highly conserved Striatin Interacting Phosphatase and Kinase (STRIPAK) complex, that antagonizes Hippo signaling. Mobs stand out for their potential to modulate the output from Hippo and Hippo-like kinases, through their roles both in activating NDR kinases and in antagonizing upstream Hippo or Hippo-like kinase activity. These opposing Mob functions suggest that they coordinate the relative activities of the Tricornered/STK38/STK38L and Warts/LATS kinases, and thus have potential to assemble nodes for pathway signaling output. We survey the different facets of Mobdependent regulation of Hippo and Hippo-like signaling and highlight open questions that hinge on unresolved aspects of Mob functions.

\section{Keywords: Mob, STE20, Hippo, NDR, Tricornered, Warts, PP2A, STRIPAK}

Abbreviations: AGC, protein kinase A, G, and C-related; Dbf2, Dumbbell former protein 2; GCK, germinal center kinase;
LATS, large tumor suppressor; MAP4K, mitogen activated protein kinase kinase kinase kinase; Mats, Mob as tumor
suppressor; MEN, mitotic exit network; Mob, Mps1 binder-related; MOR, morphogenesis Orb6 network; MST, Mammalian
STE20-like; NDR, nuclear Dbf2-related; NTR, N-terminal regulatory; PAK, p21-activated kinase; PP2A, protein phosphatase
2A; RAM, regulation of Ace2p and morphogenesis; RNAi, RNA interference; SARAH, Salvador, Rassf, and Hippo; SIN,
septation initiation network; SLMAP, sarcolemma associated protein; STE20, Sterile-20; STK, serine/threonine kinase; STRIP,
striain interacting protein; STRIPAK, striatin interacting phosphatase and kinase; WASP, Wiskott-Aldrich syndrome protein. 


\section{INTRODUCTION}

The Mps $\underline{1}$-binder-related (Mob) family of adaptor proteins is associated with both Hippo and Hippo-like signaling pathways. Mobs impact these pathways through interactions with both effector kinases and an inactivating phosphatase. Distinct classes of Mobs are associated with distinct complexes. Mobs are generally identified as kinase activators, due to their wellcharacterized ability to activate specific kinases in yeast, and to partially activate the Warts/LATS kinases (Xu et al., 1995). Warts/LATS kinases are maximally activated by Hippo kinase and are central to Hippo pathway growth control in animals. Surprisingly, Mobs are also components of a phosphatase regulatory complex known as STRIPAK, which dampens output levels for Hippo signaling (Figure 1). Altogether, Mobs appear to act as adaptors in assembling subcellular nodes that activate or inactivate Hippo. The growing network of Mobs and Mobinteracting proteins suggests that modulation of Mob levels could provide a means of controlling the availability of specific regulatory nodes within a cell or tissue (Sasaki et al., 2007; Lignitto et al., 2013; Otsubo et al., 2017; Chen et al., 2018). Regulation of Mob levels in mouse and human systems was covered recently in Gundogdu and Hergovich (2019).

The founding member of the Mob family, Mps one binder 1 (Mob1), was discovered in a yeast two hybrid screen for

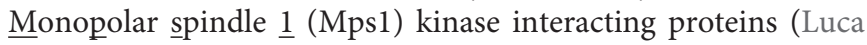
and Winey, 1998a). However, early studies of Mobs characterized them as activators of a different group of serine-threonine kinases: yeast Dumbbell former 2 (Dbf2; Komarnitsky et al., 1998) and Warts/LATS in flies and mammals (Lai et al., 2005; Hergovich et al., 2006a). Subsequently, Warts/LATS kinase and its partner Mob were defined as core constituents of the Hippo signaling pathway in flies and mammals (Hergovich, 2016; Misra and Irvine, 2018). Consistent with this function, altered regulation of, or mutations in human mob genes are associated with numerous cancers (reviewed in Sharif and Hergovich, 2018; Gundogdu and Hergovich, 2019).

The kinase-activating function of Mobs was supported by identification of a second group of fungal Dbf2-related kinases that are activated by dedicated Mob partners (Colman-Lerner et al., 2001; Hou et al., 2003; Maerz and Seiler, 2010). In parallel, orthologous kinases were identified in filamentous yeast, flies, and mice: Colonial temperature sensitive $\underline{1}(\operatorname{Cot} 1)$, Tricornered and NDR kinases, respectively (Yarden et al., 1992; Millward et al., 1995; Geng et al., 2000; Devroe et al., 2004; Hergovich et al., 2005). This second, Tricornered-like group of kinases is regulated by upstream kinases that share sequence similarity with Hippo. For this reason, we assign them to a Hippo-like kinase signaling pathway. Here, we will use "NDR kinase" as a general term to include both groups of Dbf2-related kinases.

Mps1-binder-related genes are predicted from all eukaryotes surveyed (Vitulo et al., 2007; Figure 2). Animal Mobs cluster into four classes; current evidence supports functional divergence among the four Mob classes. Only Class I Mobs are routinely designated as core components of Hippo signaling pathways (most recently in Davis and Tapon, 2019; Zheng and Pan, 2019). However, both Class I and Class II Mobs can bind NDR kinases and regulate their activity within Hippo and Hippolike pathways. Class II Mobs may have additional adaptor functions independent of NDR kinases (Gomez et al., 2015). The more divergent Class III and Class IV Mobs have attracted less attention, but this is changing. Class IV/Phocein Mobs are components of the PP2A regulatory complex known as STRIPAK and antagonize the activation of NDR kinases, as well as others. Potential Mob functions beyond Hippo and Hippo-like pathways are reviewed by Gundogdu and Hergovich (2019) and will not be discussed here.

We begin with the structural features of the Mobs, and how they differ between classes. This leads to discussion of known interactions of Class I and Class II Mobs with NDR kinases, and then with other interacting proteins involved in Hippo and Hippo-like pathway activation. We move on to summarize functions gleaned from genetic studies, first in unicellular yeast models, and then in the fly animal model. Functional studies with mouse knockout strains and human cell lines were reviewed in depth recently (Gundogdu and Hergovich, 2019), and will not be covered here. Emerging information about STRIPAK phosphatase complex regulation of Hippo signaling is discussed last.

\section{SHARED STRUCTURE AND DISTINGUISHING FEATURES BETWEEN THE FOUR CLASSES OF ANIMAL MOBS}

How the distinct functions of each Mob class are related to their sequence divergence is poorly understood. Even the Mob family name implies a distinct function, through the reference to Mps1 kinase (Luca and Winey, 1998a), which acts in the spindle assembly checkpoint (Liu and Winey, 2012; London and Biggins, 2014). Some NDR kinases and Mobs have been implicated in spindle orientation (Frenz et al., 2000; Trammell et al., 2008; Chiba et al., 2009; Dewey et al., 2015; Yan et al., 2015), but the significance of binding interactions with Mps1 kinase remains unclear.

Generally, Mobs are single domain proteins, with an average length of 210-240 amino acids (Hergovich, 2011; Gundogdu and Hergovich, 2019). The Mob/Phocein domain adopts a conserved globular fold, based on solved structures of three classes of Mobs from multiple species: Class I Mobs from Homo sapiens, Xenopus laevis, Mus musculus, and Saccharomyces cerevisiae, a Class II Mob from S. cerevisiae, and a Class IV Mob from H. sapiens (Stavridi et al., 2003; Ponchon et al., 2004; Mrkobrada et al., 2006; Rock et al., 2013; Chung et al., 2014; Gógl et al., 2015; Ni et al., 2015; Kim et al., 2016; Couzens et al., 2017; Kulaberoglu et al., 2017; Xiong et al., 2017; Chen et al., 2018; Parker et al., 2019). This conserved structure is the Mob family fold (Figure 2B). The Mob family fold forms the NDR kinase binding surface for Class I and Class II Mobs. Whether Class III Mobs retain this tertiary structure is an open question.

Although the shared Mob/Phocein domain suggests that all Mobs might bind to NDR kinases, the evidence does not support this notion. Binding between NDR kinases and Class III or IV Mobs was undetectable in multiple independent assays 


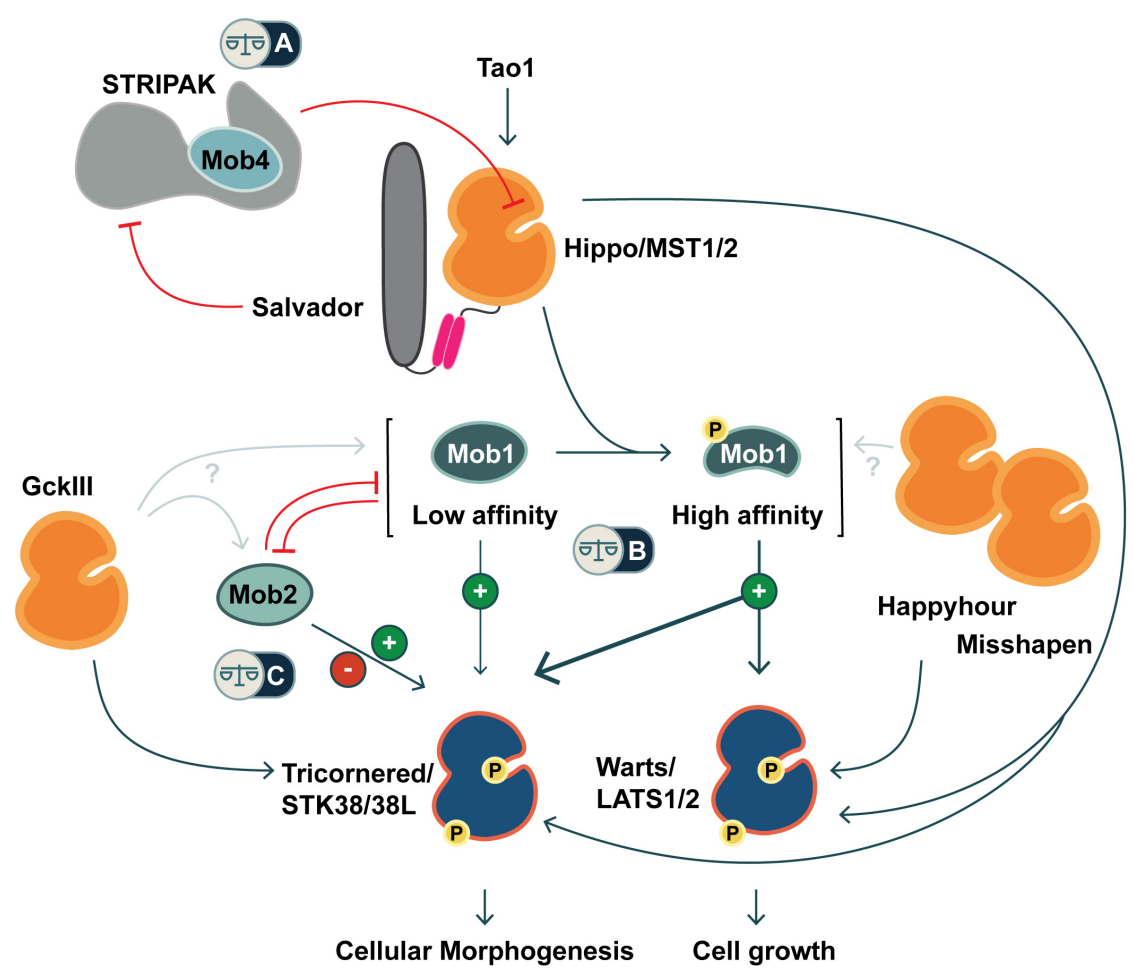

FIGURE 1 | Co-regulation of Hippo and Hippo-like signaling pathways by Mob family proteins. Metazoans are equipped with two classes of NDR kinases, Tricornered- and Warts/LATS kinases. Mob family proteins have potential to balance the activities of these two classes of kinases in three ways (noted by balance icons). This pathway schematic is based on evidence from fly and mammalian literature, as discussed in the text. (A) As a STRIPAK component, Mob4/Phocein antagonizes Hippo/MST1/2 kinase activation. In balance with Salvador/Sav1 and Tao1, Mob4/Phocein determines Hippo/MST1/2 kinase activity. (B) The phosphorylation status of Class I Mob proteins alters their affinity for their NDR kinase partners. Non-phosphorylated Mob1 binds a human Tricornered-like kinase (STK38/STK38L) but not the Warts/LATS kinase (LATS1/2). Upon phosphorylation, Mob1 undergoes an activating allosteric transition that results in increased affinity for both Warts and Tricornered classes of NDR kinases. (C) Tricornered and Tricornered-like kinases bind both Class I and II Mob proteins. The effect of Mob2-binding to Tricornered-like kinases is unclear, with reports ascribing activating as well as inhibitory roles. Class II Mobs compete with Class I Mobs for binding to Tricornered-like kinases. In addition to Hippo, other STE20 kinases function upstream of NDR family kinases (Happyhour/MAP4K3, Misshapen/TNIK, GckIII/MST3/4), but it is not known whether they directly phosphorylate Mob family proteins.

(Maerz et al., 2009; Kohler et al., 2010; Ribeiro et al., 2010; Kwon et al., 2013; Xiong et al., 2017). Furthermore, NDR kinases were absent from binding partners identified both for fly Mob4 and all human Class III and IV Mobs, through proteomic surveys (Ribeiro et al., 2010; Xiong et al., 2017). Class III and IV Mobs appear to lack the capacity for stable binding to NDR kinases. In contrast, Class III and IV Mobs can physically associate with Hippo and Hippo-like kinases, in some cases as part of a STRIPAK complex. Thus, Class III and IV Mobs also contribute to regulation of Hippo signaling (Tang et al., 2014).

\section{MOBS AS COMPONENTS OF NDR KINASE ACTIVATION COMPLEXES}

Many Mob gene sequences are labeled with a "kinase activator" function, due to their shared Mob/Phocein domain. This functional tag poorly represents the growing range of interactions between Mobs and other core Hippo and Hippo-like pathway core components (Mah et al., 2001; Bichsel et al., 2004;
Devroe et al., 2004; Lai et al., 2005; Rock et al., 2013; Gógl et al., 2015; Xiong et al., 2018).

\section{Direct Mob-NDR Kinase Interactions}

The kinase activator tag for Mob genes arises from their essential functions in signaling pathways involving NDR serine-threonine kinases, which are core components of Hippo and Hippolike signaling pathways. Phosphorylation of NDR kinases by Hippo and Hippo-like kinases creates a short kinase cascade to activate downstream effectors of the pathways (Hergovich, 2013; Hergovich, 2016). At least two NDR kinase genes have been identified in sequenced eukaryote genomes, including both unicellular and multicellular fungi (Johnston and Thomas, 1982; Johnston et al., 1990; Toyn et al., 1991; Yarden et al., 1992). Plant NDR kinases are more divergent compared to those of fungi and animals, with potential for greater functional diversity, but little is known about Mob interactions with NDR kinases in plants (Kameshita et al., 2010; Katayama et al., 2012; Rademacher and Offringa, 2012; Zermiani et al., 2015; Cui et al., 2016; Xiong et al., 2016). Yeast studies have been foundational for the assignment of Mobs as NDR kinase activators. 
A

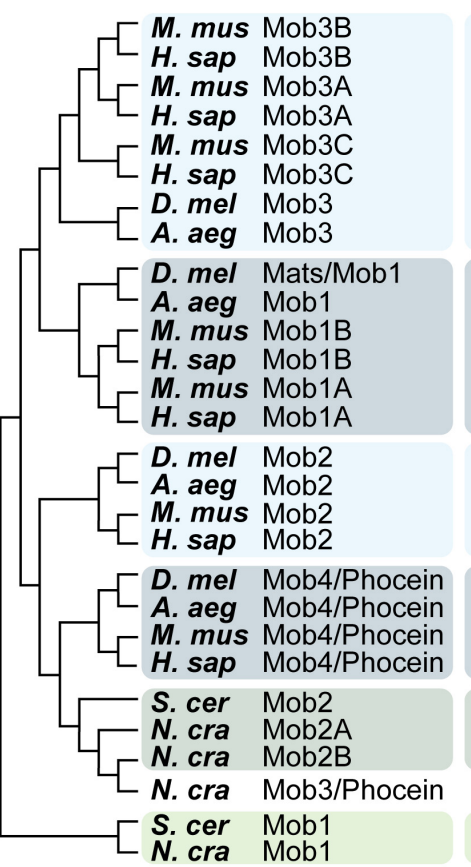

B

\section{Human Mob1A (PDB: 4JIZ)}

STE20 kinase binding surface

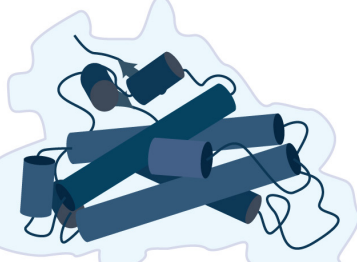

NDR kinase binding surface

Percent Identity Matrix

D. mel

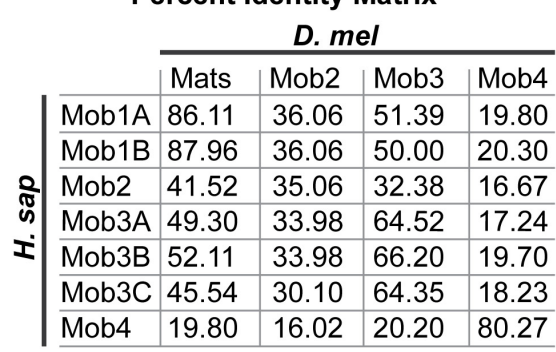

FIGURE 2 | Mob family proteins are conserved from yeast to humans. (A) Molecular phylogenetic analysis of Mob protein sequences from the indicated species. Tree was generated using MEGA X (Jones et al., 1992; Kumar et al., 2018) as described by Hall (2013). Animal Mob proteins cluster into four distinct classes (I-IV). The relative divergence between these classes is not revealed in this limited comparison of Mobs from four animal and three fungal species - three fungal Mob classes are indicated (FI-FIII). For a thorough discussion of the evolutionary history of Mob family proteins consult the work of Vitulo et al. (2007) and Ye et al. (2009). A. aeg, Aedes aegypti; D. mel, Drosophila melanogaster; H. sap, Homo sapiens; M. mus, Mus musculus; N. cra, Neurospora crassa; S. cer, Saccharomyces cerevisiae. Refer to the Pfam database which maintains a manually curated annotation of the Mob family proteins (PF03637; El-Gebali et al., 2019). Mob family protein sequences are readily available at uniprot.org by searching for "Mob kinase activator" (The UniProt Consortium, 2019). (B) Artistic sketch of the human Mob1A protein (PDB: 4JIZ) reported by Rock et al. (2013). Mob proteins adopt a conserved globular fold with a core that consists of a four alpha-helix bundle, we refer to this fold as the "Mob family fold." Binding to NDR kinases or STE20 kinases takes place on distinct Mob surfaces as indicated. (C) Percent identity matrix between human and fly Mob proteins.

\section{NDR Kinase Domains Involved in Activation and Mob Interactions}

Nuclear Dbf2-related kinases are members of the AGC serinethreonine protein kinase family (Hanks and Hunter, 1995; reviewed in Pearce et al., 2010; Rademacher and Offringa, 2012; Arencibia et al., 2013). Like other AGC kinases, NDR kinase activity depends on phosphorylation at two sites: the activation segment, which is conserved in all eukaryotic protein kinases, and the C-terminal hydrophobic motif (HM), which is shared by most AGC kinases. NDR kinases are distinguished by two defining features (Figure 3). The first is an extended activation segment (Millward et al., 1995; Bichsel et al., 2004), which auto-inhibits the kinase when unphosphorylated. Human NDR kinase STK38 (also called NDR1) shows increased kinase activity when that segment is mutated or deleted (Bichsel et al., 2004; Xiong et al., 2018).

The second distinguishing feature of NDR kinases is the NTR region: a structural domain that functions as the Mobbinding surface (Gógl et al., 2015; Ni et al., 2015; Kim et al., 2016; Kulaberoglu et al., 2017; Parker et al., 2019). Each of the yeast NDR kinases binds to a specific Mob that is necessary, but insufficient, for kinase activity (Komarnitsky et al., 1998; Luca and Winey, 1998a; Frenz et al., 2000; Mah et al., 2001; ColmanLerner et al., 2001; Hou et al., 2003). The current model is that
Mob binding alters the NDR kinase conformation (Gógl et al., 2015; Manning and Harvey, 2015; Vrabioiu and Struhl, 2015; Xiong et al., 2018).

Altogether, the prevailing model is that at least three regulatory inputs are required to maximally activate an NDR kinase (Figure 3). First, the NDR kinase is weakly activated by binding to a Mob. Second, the NDR kinase is phosphorylated at a conserved, C-terminal HM by Hippo or a Hippo-like kinase. Third, the weakly active NDR kinase becomes fully activated through auto-phosphorylation (reviewed in Hergovich et al., 2006b; Hergovich, 2016).

\section{Class I and II Mobs Bind NDR Kinases Through Overlapping but Distinct Amino Acids}

The single-celled yeasts S. cerevisiae and S. pombe each encode two classes of mob genes and each Mob protein class has a dedicated NDR kinase binding partner. For fungi and animals, NDR kinases can be subdivided into either the Warts/LATS or the Tricornered-like class, based on their kinase domain sequence. Unlike this specificity in yeast Mob-NDR kinase binding, animal Class I Mobs are promiscuous in their association with both types of NDR kinases. For example, 


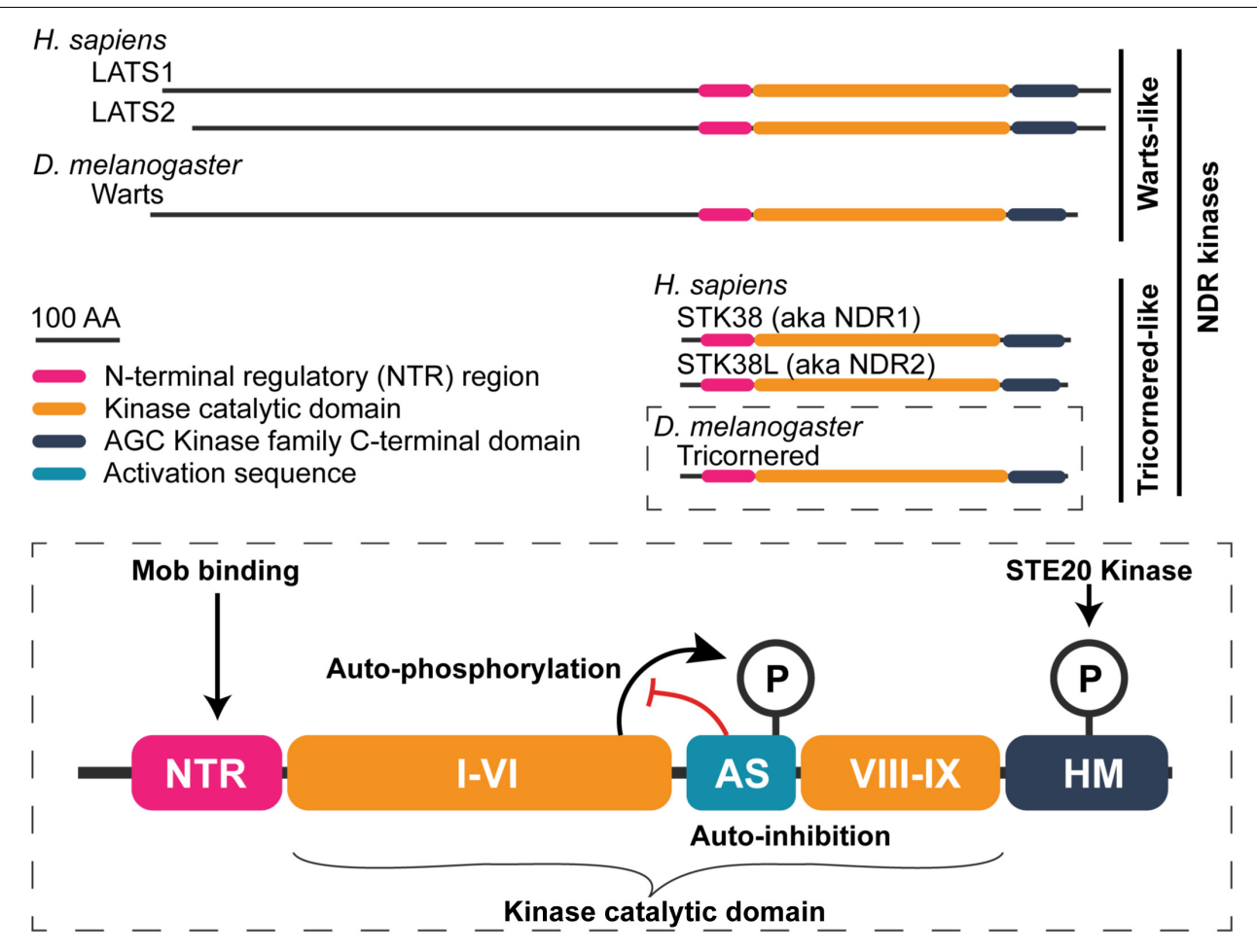

FIGURE 3 | General structure of nuclear Dbf2-related (NDR) family kinases. Schematic of human and fly NDR kinases. Warts/LATS NDR kinases have a N-terminally extend domain relative to the shorter Tricornered-like kinases. The elongated $\mathrm{N}$-terminus includes additional protein-protein interaction motifs (see Furth and Aylon, 2017). A detailed view of the Tricornered kinases is shown in the bottom to highlight structural features that are conserved among the NDR kinases. NDR kinases contain an NTR region to which Mob proteins bind. Within the kinase catalytic domain, between sub-domains VI and VIII, NDR kinases contain a long activation segment (AS) with auto-inhibitory function ( 30-60 amino acids in length). NDR kinase auto-phosphorylation at the AS counteracts its auto-inhibitory function. Lastly, NDR family kinases possess a hydrophobic motif (HM) in their C-terminus. Phosphorylation of the HM is essential for full kinase activation. STE20 family kinases (e.g., Hippo, Misshapen, and Happyhour in Drosophila) phosphorylate this motif.

the fly Class I Mob, Mats, can physically associate with each of the fly NDR kinases: Warts/LATS and Tricornered (He et al., 2005a). Similarly, mammalian Class I Mobs can bind to either Warts/LATS or Tricornered-like kinases: LATS1/2 and STK38/STK38L, respectively (Bichsel et al., 2004; Devroe et al., 2004; Kohler et al., 2010; Kulaberoglu et al., 2017; Xiong et al., 2017). Partnering dynamics between a Class I Mob and an NDR kinase are conditional and selective, based on structure-function studies of murine and human Class I Mobs bound to the NTR of either a Warts/LATS or Tricornered-like kinase (Ni et al., 2015; Kim et al., 2016; Kulaberoglu et al., 2017).

Phosphorylation of Class I Mobs modulates their binding affinity for different partners (Figure 1). For example, phosphorylation of human Mob1A is necessary for detectable association with the NTR of human LATS1 kinase (Kulaberoglu et al., 2017; see also Praskova et al., 2008; Ni et al., 2015; Kim et al., 2016). In contrast, the NTR of the Tricornered-like kinase, STK38L, will readily bind to an unphosphorylated, N-terminally truncated Mob1A (Mob1A $\left.{ }^{33-216}\right)$ in isothermal titration calorimetry assays. However, the binding affinity is significantly increased when Mob1 $\mathrm{A}^{33-216}$ is phosphorylated.

These contrasting observations may be explained by differential availability of binding sites on Class I Mobs, determined through structural studies and sequence comparisons. The NDR kinase binding surface is only partially exposed in unphosphorylated Class I Mobs (Mrkobrada et al., 2006; Ni et al., 2015; Kim et al., 2016). Full exposure of the NDR kinase binding surface is triggered by phosphorylation of highly conserved $\mathrm{N}$-terminal threonine residues, through a conformational change in Class I Mobs. The importance of a phosphorylation-induced conformation change may come from the overlapping but distinct sets of amino acids that mediate binding between Mob1 and Warts/LATS kinases versus Tricornered-like kinases.

The critical amino acids have been identified for formation of mammalian Mob1-NDR kinase complexes (Kohler et al., 2010; Ni et al., 2015; Kim et al., 2016; Kulaberoglu et al., 2017). How specific amino acid contacts contribute to binding specificity was tested for a Class I Mob. One critical interaction involves a conserved histidine in Warts/LATS kinases, which forms a hydrogen bond with a class I Mob (Kulaberoglu et al., 2017). This histidine is replaced by either a phenylalanine or a tyrosine in the corresponding position of Tricorneredlike kinases in yeast, flies, and humans. For human Mob1A binding to human Warts/LATS kinase, LATS1, this bond occurs between aspartic acid-63 (D63) of Mob1A and histidine-646 of LATS1. When Mob1A-D63 is replaced by a non-polar residue, valine, the resultant Mob1A-D63V mutant protein fails to bind 
the NTR of either fly or mammalian Warts/LATS kinases. However, Mob1A-D63V retains the ability to bind the NTR of Tricornered-like kinases, as assayed by immunoprecipitation from either fly or mammalian cells. Thus, animal Class I Mobs make distinct binding contacts with each type of NDRkinase. The potential for differential exposure of binding sites may explain the differential binding between unphosphorylated Mob1A with either Warts/LATS kinases or Tricorneredlike kinases.

Class II Mobs also have demonstrated functions as co-factors for the Tricornered-like NDR family kinases. Several studies have demonstrated this association for human Class II Mobs (Devroe et al., 2004; Chiba et al., 2009; Kohler et al., 2010; Xiong et al., 2017). A supporting result comes from an observation of Class II Mob-NDR kinase interactions in flies (He et al., 2005b). However, the functional consequences for Class II Mob binding to human Tricornered-like kinases are controversial.

Initial studies proposed that human Class II Mobs were Tricornered-like kinase co-activators (Devroe et al., 2004; Chiba et al., 2009). Devroe et al. (2004) provide three lines of evidence to support a co-activator function. First, human Mob2 directly binds human STK38/STK38L in a co-immunoprecipitation assay from 293T cells. Second, Mob2 and STK38/STK38L partly colocalize when expressed in HeLa cells. Finally, Mob2 stimulates STK38/STK38L autophosphorylation in vitro, in kinase assays. Consistent with these findings, Chiba et al. (2009) showed that forced expression of Mob2 in HeLa cells increases STK38 activity 5.4-fold relative to controls.

Contrasting results come from a subsequent report, where three lines of evidence suggest that human Mob2 inhibits the activity of the human Tricornered-like kinases. In this case, Mob2 antagonism is detected through competitive interactions with Class I Mobs. Kohler et al. (2010) found that Mob2 can outcompete Mob1A for STK38 binding, when assayed by immunoprecipitation from HEK293 cells (Figure 1). Strikingly, they show that Mob2 knock-down by RNAi leads to increased levels of activated STK38L, suggesting an inhibitory function. Additional experiments suggest that Mob2 can antagonize STK38/STK38L activation when Mob1A is modified with a membrane-targeting sequence in COS-7 cells. The role of Mob2 as a competitive antagonist of Mob1A remains to be tested by a comparison that uses wild-type Mob1A.

Overall, more work is needed to reconcile these opposing models for the function of Mob2 in regulating Tricornered-like kinases. It will be valuable to determine whether different cellular contexts may influence the outcomes of Class II Mob binding to Tricornered-like NDR kinases. A better understanding of the structural features and amino acid contacts that are involved in direct binding between Class II Mobs and animal Tricorneredlike kinases could also be useful.

\section{Potential for Binding Competition Between Mobs and Other NDR Kinase Binding Proteins}

For both Warts/LATS and Tricornered-like kinases, alternative binding complexes that lack Mobs have been reported. How such
Mob-deficient complexes impact NDR kinase activity is emerging for Warts/LATS kinases.

\section{Competition Between Mob1 and LIM Proteins for Binding to Warts/LATS Kinases}

In animals, the Warts/LATS kinase N-terminus is separated from the Mob-binding NTR domain by an N-terminal extension; this domain is substantially elongated compared to the N-terminus of Tricornered-like kinases (Figure 3). Proper Warts/LATS kinase function requires the N-terminal extension, which mediates interactions with both positive and negative regulators (Yabuta et al., 2013; Furth and Aylon, 2017). Recruitment of Warts/LATS kinases to the cell membrane requires the $\mathrm{N}$-terminal extension in flies and humans, primarily through association with one of several proteins: Expanded, Merlin/NF2, and Ajuba (Hamaratoglu et al., 2005; Das Thakur et al., 2010; Yin et al., 2013; Rauskolb et al., 2014; Meserve and Duronio, 2015; Yu et al., 2015; Su et al., 2017; Ibar et al., 2018). The potential for competition between Mob1 and other regulators with overlapping binding domains has been uncovered for Warts/LATS kinase association with the LIM proteins, fly Ajuba, and human TRIP6.

Ajuba inhibition of Warts/LATS kinase activity was studied in epithelial cells of fly wing primordia. Inactive Warts/LATS is enriched at adherens junctions, where it binds to its inhibitor, Ajuba (Das Thakur et al., 2010; Rauskolb et al., 2014; Meserve and Duronio, 2015). Active Warts/LATS resides more apically, where it binds to Expanded and Merlin/NF2 complexes (Hamaratoglu et al., 2005; Yin et al., 2013; Sun et al., 2015; Yu et al., 2015; Su et al., 2017). Fly Mob1 (Mats) is required for the release of Warts/LATS from Ajuba inhibition at the adherens junction and association of active Warts/LATS with more apical Expanded. The mechanism for relocation is unknown, but it requires additional proteins involved in Warts/LATS kinase activation: the scaffold protein Salvador and Hippo kinase (Sun et al., 2015).

A potential mechanism for the inhibition of Warts/LATS kinase activity comes from studies of the human LIM protein, TRIP6. Dutta et al. (2018) found that TRIP6 antagonizes LATS1/2 kinases through a direct competition with Mob1A. TRIP6 binds to the N-terminal extension at a site that overlaps the Mob binding domain of LATS1/2 kinases. LATS1/2 forms mutually exclusive complexes with either TRIP6 or Mob1, as determined by competitive binding assays. Furthermore, LATS1/2 kinase activity increased upon TRIP6 knock-out in HEK293 cells. Thus, TRIP6 antagonizes LATS1/2 activity by blocking kinase association with an activating Class I Mob.

Altogether, these studies suggest that competition between the LIM proteins, Ajuba, and TRIM6, and Class I Mobs mediates both membrane domain localization and activity of Warts/LATS kinases in both flies and human cells. However, open questions remain about the mechanisms. Does Mob binding simply release Warts/LATS kinase from the adherens junction, permitting Warts/LATS association with other Hippo pathway components in a more apical domain? Alternatively, does Class I Mob-binding have a direct role in recruiting Warts/LATS kinase to complexes at the apical membrane? 


\section{Evidence for Mutually Exclusive Mob1- and Beclin1-Complexes With a Human Tricornered-Like Kinase}

The Mob-binding NTR domain of Tricornered-like kinases is close to the $\mathrm{N}$-terminus. The absence of an $\mathrm{N}$-terminally extended sequence indicates that Tricornered-like kinases are regulated in distinct ways from the Warts/LATS class. Consistent with this, Tricornered-like kinases function in autophagic pathways is associated with specific protein partners, identified through yeast-two hybrid assays (Joffre et al., 2015; Joffre et al., 2016), immunoprecipitation-mass spectrometry analysis (Klimek et al., 2019), and proximity labeling followed by mass spectrometry (Martin et al., 2019).

Beclin1 is a Tricornered-like kinase binding partner and a key regulator of autophagy. Like the Mobs, Beclin1 binds to the NTR region of the human STK38 Tricornered-like kinase. Surprisingly, both Mob1 and Beclin1 are required for STK38 function in autophagy (Joffre et al., 2015). These authors propose that STK38 binds to either Mob1 or Beclin1, but that both types of complexes have functions in autophagy. It is unclear whether Mob1 and Beclin1 compete for STK38 binding or whether partner selection is otherwise regulated. Whether other Tricornered-like kinase binding-partners can target kinase function for specific biological processes is an open question.

\section{Mob Proteins Interact With STE20 Kinases to Regulate NDR Kinase Activity}

Hippo kinase activation of Mob-NDR kinase partners parallels the STErile20 (STE20) kinase regulation of Mob-NDR kinases, initially elucidated in yeasts. Fly Hippo kinase is a member of the STE20 kinase family, which is grouped together based on sequence similarity in their kinase domain, and on conservation of an auto-phosphorylation consensus sequence that is essential for their activation (Ling et al., 2008; Record et al., 2010). The large STE20 family is divided into

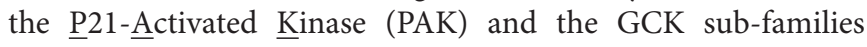
(Dan et al., 2001; Record et al., 2010; Miller et al., 2019; reviewed in Thompson and Sahai, 2015). Only the GCK sub-family kinases have been implicated in Mob or NDR kinase function.

The mouse and human orthologs of Hippo kinase are the Mammalian STerile20-like kinases $\underline{1}$ and $\underline{2}$ (MST1/2). MST1 and MST2 can activate NDR kinases and have the highest sequence similarity scores to Hippo (FlyBase FB2020_01; Thurmond et al., 2019). We group them together as the Hippo kinases here. Other mammalian NDR kinase activating kinases include GckIII kinases (Stegert et al., 2005; Poon et al., 2018), MAP4Ks (Li et al., 2014, 2015; Meng et al., 2015; Zheng et al., 2015) and TAO kinases 1 and 3 (Plouffe et al., 2016). In flies, GckIII and two MAP4Krelated kinases (Happyhour and Misshapen) can activate an NDR kinase (Figures 1, 4A; Li et al., 2014; Zheng et al., 2015; Poon et al., 2018). It is not clear whether MAP4K activation of NDR kinases requires an associated Mob [see Zheng and Pan (2019) for discussion].

\section{Mob Proteins Interact With Hippo Kinases to Regulate NDR Kinase Activity}

When activated, Hippo kinases phosphorylate Class I Mob proteins, which alters the Mob's specificity and affinity for NDR kinases (Figure 1). Hippo kinases also directly activate NDR kinases, through phosphorylation of NDR kinase HMs (Wei et al., 2007; Ni et al., 2015; Kim et al., 2016; Kulaberoglu et al., 2017; Xiong et al., 2017). In flies and mammals, Class I Mobs were initially proposed to be adaptor proteins that are required for Warts/LATS kinase activation through phosphorylation by Hippo kinases.

Mobs can directly bind to Hippo kinases in both flies and mammals. The Mob/Phocein domain has a phospho-peptide binding pocket that binds to specific phosphorylated threonines in Hippo kinase linker regions (Ni et al., 2015; Couzens et al., 2017; Xiong et al., 2017; see also Rock et al., 2013) for the analogous binding mechanism in budding yeast, S. pombe]. These linker residues are autophosphorylated by both Hippo and Hippo-related kinases, such as the mammalian GckIII ortholog, MST4 (Ni et al., 2015; Chen et al., 2018). Thus, Mob binding to Hippo kinases is promoted by autophosphorylation.

Mob-driven assembly of Class I Mob-NDR kinase-Hippo kinase ternary complexes is thought to be a critical step in NDR kinase activation. The Mob structure could facilitate complex formation, in that the phospho-Hippo binding pocket of a Class I Mob is located opposite to the NDR kinase binding surface (Figure 2B; Kim et al., 2016). This structural arrangement would link Hippo kinases to both phosphorylation substrates: The Mob and its NDR kinase binding partner (Figure 5A; $\mathrm{Ni}$ et al., 2015). In support of this view, deletion of the Mob-binding linker region of the human Hippo kinase, MST2, blocks it from phosphorylating Mob1.

Whether other Hippo-related kinases bind to or phosphorylate Mobs is an open question. NDR kinases can be activated by MAP4K- and GckIII-group GCK kinases, but it is unknown whether Mobs are involved (Gundogdu and Hergovich, 2019; Zheng and Pan, 2019). It remains unclear whether Mobs can generally recognize and bind to these Hippo-related kinases through phospho-threonines in the kinase linker domains.

One study has raised the question of whether stable Mob1Hippo kinase binding is essential for Mob1 phosphorylation or Warts/LATS kinase activation by Hippo kinases (Kulaberoglu et al., 2017). To test the requirement for Class I Mobs in Hippo kinase binding to its substrate NDR kinase, Kulaberoglu et al. (2017) generated a human Mob1A ${ }^{\mathrm{K} 104 \mathrm{E} / \mathrm{K} 105 \mathrm{E}}$ mutant that fails to bind fly or human Hippo kinases. However, Mob1A ${ }^{K 104 E / K 105 E}$ retains the ability to bind to either class of NDR kinase when assayed by co-immunoprecipitation in human or fly cells. Expression of Mob1A ${ }^{\text {K104E/K105E }}$ rescues lethality in mats mutant flies, which lack the single Class I Mob, and restores normal expression of Warts target genes in fly wing primordia. These results suggest that STE20 family kinases that lack phospho-peptide motifs recognized by Mobs may still contribute to NDR kinase activation. 

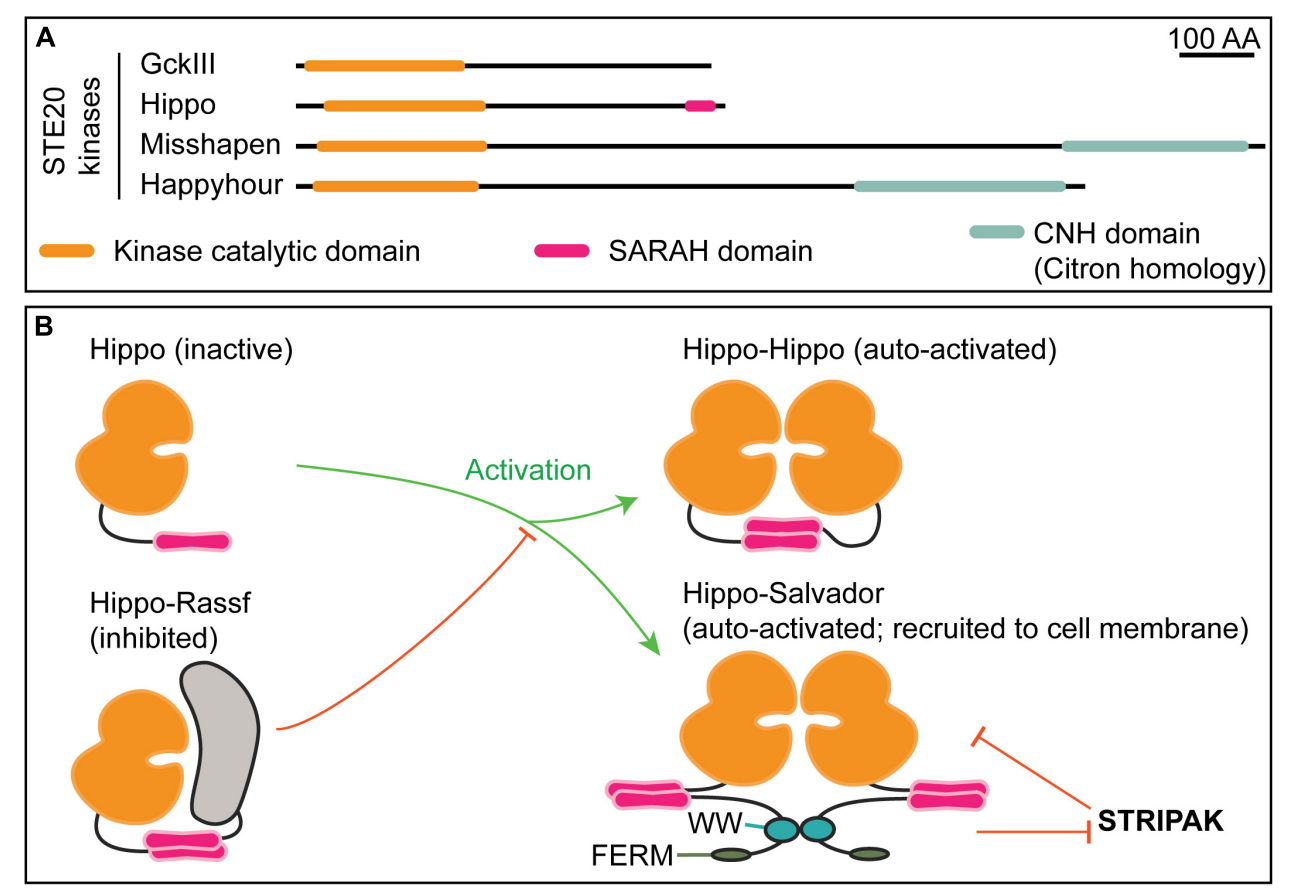

FIGURE 4 | STE20 kinases have distinct domain architectures and regulatory strategies. (A) Schematic of select Drosophila STE20 kinases that have been demonstrated to phosphorylate NDR kinases. The depicted domain organization is based on annotation by UniProtKB/TrEMBL (The UniProt Consortium, 2019); the accession numbers are: Q9VEN3 (GckllI), Q8T0S6 (Hippo), Q9W002 (Misshapen), and A1ZBH7 (Happyhour). Note that Hippo is the sole STE20 kinase with an annotated SARAH (Salvador, Rassf, Hippo) domain. The function of the Citron homology domain (CNH) in NDR kinase regulation is not known. (B) Mutually exclusive SARAH-SARAH binding interactions provide the structural basis for positive and negative regulation of Hippo kinase activity. Formation of Hippo-Rassf antagonizes assembly of Hippo-Hippo or Hippo-Salvador complexes both of which promote full-activation via Hippo trans-autophosphorylation. When in a complex with Salvador, Hippo may be refractive to STRIPAK-mediated inactivation (Bae and Luo, 2018).

The possibility that Mobs can be bypassed in Hippo activation of NDR kinases might indicate that recruitment of Hippo-like kinases and Warts-Mob1 complexes to overlapping sub-cellular domains is sufficient to overcome the loss of stable Mob1Hippo-like kinase binding (as presented in Yin et al., 2013; Su et al., 2017). Alternatively, brief, or unstable interactions between Mob1A $\mathrm{A}^{\mathrm{K} 104 \mathrm{E} / \mathrm{K} 105 \mathrm{E}}$ and Hippo kinase may permit sufficient Warts kinase activity for pathway responses in vivo, even though such binding cannot be detected by standard assays. More work is needed to reconcile distinct interpretations for Class I Mob function in NDR activation complex assembly (compare models proposed by Kulaberoglu et al., 2017; Manning and Harvey, 2015; Ni et al., 2015; Vrabioiu and Struhl, 2015; Bae and Luo, 2018; Zheng and Pan, 2019).

Moving beyond Class I Mobs, all human Mobs, except Mob2, bind to a MST1-derived phospho-peptide sequence (Couzens et al., 2017); and share analogous phospho-binding pockets. Binding relies on three phosphate-coordinating basic residues [lys-153, arg-154 and arg-157 in human Mob1A, which are not conserved in human Mob2 (Rock et al., 2013; Ni et al., 2015; Couzens et al., 2017)]. Furthermore, human Mob4 binds the GCK-III STE20 kinase, MST4, through a binding interface that is similar to the human Mob1-MST1 complex; binding is also dependent on MST4 linker phosphorylation (Chen et al., 2018). Taken together, these findings suggest that most classes of Mobs are general STE20 kinase-binders. However, the Class III and IV Mobs appear not to bind NDR kinases (Kohler et al., 2010; Ribeiro et al., 2010; Kwon et al., 2013; Xiong et al., 2017), raising questions about the functions of Class III or Class IV Mob interactions with a STE20 kinase.

Human Mob4 may contribute to Warts/LATS kinase regulation indirectly by blocking Mob1 association with Hippo kinase (Chen et al., 2018). Mob4 outcompetes Mob1, in a dose-dependent manner, for binding to the human Hippo kinase, MST1 in a co-immunoprecipitation assay from PANC-1 cells. More work is needed to critically assess the possibility that Mobs contribute to STE20 signaling output through regulation of phosphorylation-target selection and/or sub-cellular localization. Additional scaffold proteins are involved in animal NDR kinase activation; they are briefly surveyed in the next section.

One feature that distinguishes Hippo kinases from other GCK kinases in the STE20 family is a SARAH domain, named after three proteins that have it: SARAH (Scheel and Hofmann, 2003). Hippo kinases bind their regulatory partners, Salvador and Rassf family proteins, through SARAH-SARAH domain binding (Sanchez-Sanz et al., 2016; Bae et al., 2017; Bae and Luo, 2018; Cairns et al., 2018). In flies, Salvador-Hippo binding leads to kinase activation (Figure 4B), whereas Rassf-Hippo binding results in kinase inhibition (Polesello et al., 2006). Thus, the SARAH domain provides one structural basis for regulation of 


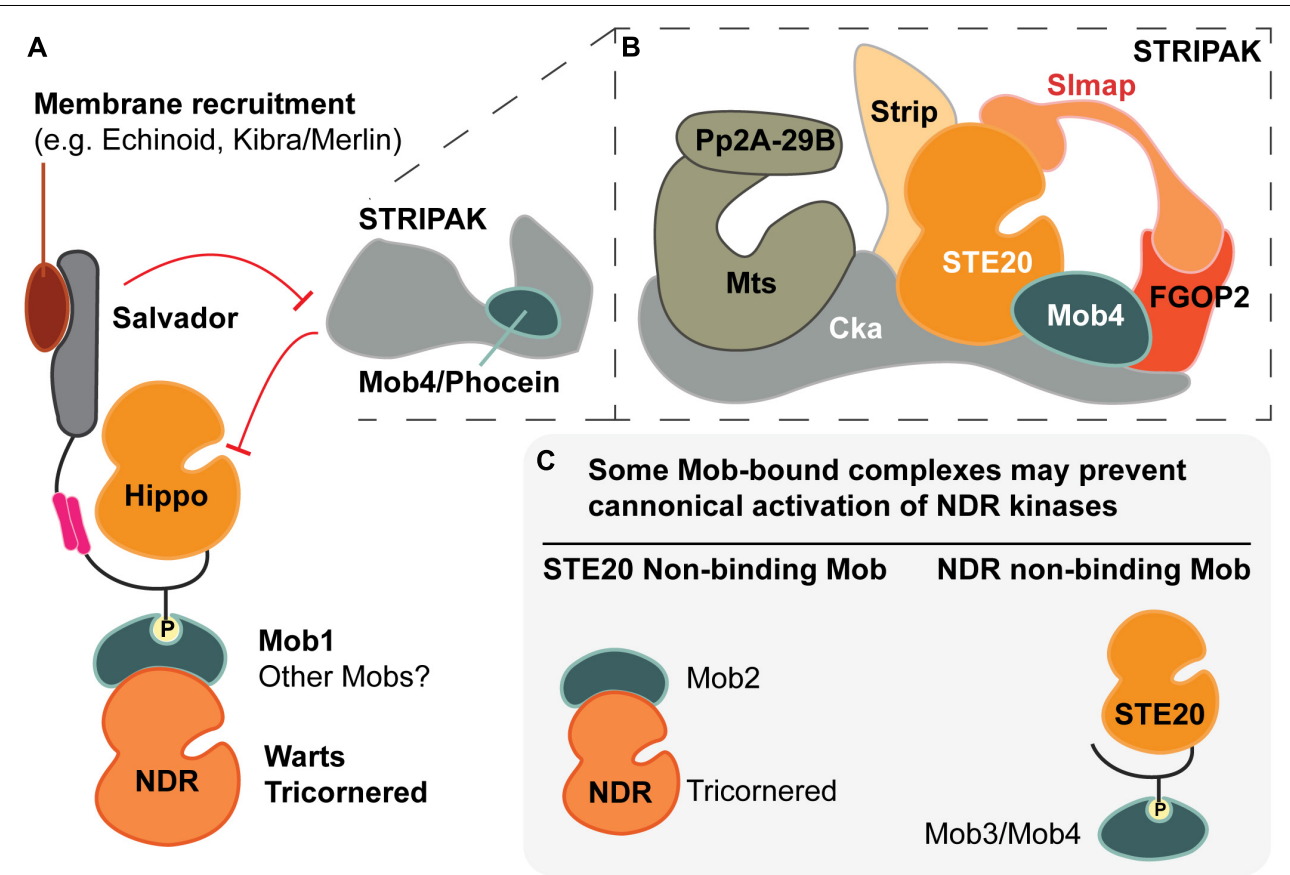

FIGURE 5 | The many facets of Mob regulatory function. (A) Metazoan Mob1 proteins have well-established roles as allosteric co-activators of NDR kinases and as scaffold proteins that bridge STE20 kinases (e.g., Hippo) to their phosphorylation targets, the NDR kinases. Together with other scaffolds, such as Salvador, these activation complexes are recruited to specific subcellular locations. In canonical Hippo signaling, the PP2A phosphatase of the STRIPAK complex inhibits Hippo via dephosphorylation. The most divergent fly Mob (Mob4/Phocein) is required for STRIPAK activity. (B) Cartoon of the STRIPAK complex adapted from Ribeiro et al. (2010), Zheng et al. (2017) and Tang et al. (2019). (C) Speculative Mob-dependent strategies of NDR kinase signaling output. Some classes of Mob proteins appear to bind STE20- or NDR-kinase binders, but not both. Such interactions yield Mob-bound complexes that may not allow formation of canonical activation complexes as pictured in part (A).

Hippo kinase activity. The regulatory features that control the activity of other GCK kinases in NDR kinase activation are less understood.

\section{Both Mobs and Scaffold Proteins Contribute to Pathway Activation Through Distinct Interactions in Different Sub-Cellular Domains}

The NDR kinase name includes "Nuclear" due to an initial observation of nuclear localization when the human Dbf2related kinase, STK38, was over-expressed (Millward et al., 1995). However, both Warts/LATS and Tricornered-like kinases move between nuclear, cytoplasmic, and/or cortical locations (Devroe et al., 2004; Emoto et al., 2004; He et al., 2005b; Hergovich et al., 2005; Horne-Badovinac et al., 2012; Yin et al., 2013; Sun et al., 2015). It is now appreciated that NDR kinase subcellular localization is tightly regulated and linked to signaling output of Hippo and Hippo-like pathways.

Early studies pointed to Mobs as regulators of human NDR kinase localization (Hergovich et al., 2005, 2006a). When human Mob1 is targeted to the membrane, both Tricornered-like and Warts/LATS kinases are localized to the membrane and potently activated (Hergovich et al., 2005, 2006a), a result reproduced in flies (Ho et al., 2010). We previously discussed a potential role for Mob1 in subcellular localization of Warts/LATS. Whether animal Mobs generally target NDR kinases to specific subcellular locations, as has been documented in yeast (Rock et al., 2013), is still unclear.

In flies and mammals, Salvador and Furry family proteins have been characterized as scaffolds that play essential roles in NDR kinase activation (Cong et al., 2001; Kango-Singh et al., 2002; Tapon et al., 2002; Chan et al., 2005; Chiba et al., 2009). Furry-related proteins are conserved from yeast to humans, while Salvador family proteins appear to lack clear homologs in yeast (Gruneberg et al., 2001; Du and Novick, 2002; Nelson et al., 2003; Rock et al., 2013; Nagai and Mizuno, 2014). We will focus on the animal scaffolds in this section, to emphasize contextdependent regulatory interactions during assembly of the NDR kinase activation complex. Notably, Salvador and Furry proteins, together with Mobs, promote the assembly of animal Hippo and Tricornered-like kinase activation complexes in specific subcellular locations.

\section{Salvador Proteins Promote Activation of Animal Warts/LATS Family Kinases}

Salvador proteins are required for the activation of Warts/LATS NDR kinases in vivo (Kango-Singh et al., 2002; Tapon et al., 2002; Pantalacci et al., 2003), and are considered core components of Hippo signaling. Activation of Warts/LATS kinases occurs near the plasma membrane; thus, subcellular localization of Warts/LATS NDR kinases and other activation complex 
components provides a potential step for pathway regulation. Substantial evidence supports the role of Salvador in recruitment of Hippo and MST1/2 kinases to specific domains of the plasma membrane. The emerging model hinges on Hippo/MST1/2 activation and presents a multi-functional view of Salvador function. On the one hand, Salvador promotes Hippo/MST1/2 activation via kinase auto-phosphorylation (Ni et al., 2013; Bae et al., 2017). On the other hand, Salvador antagonizes negative regulators of Hippo/MST1/2 kinase activity (Bae et al., 2017).

Salvador proteins are non-catalytic, with three defining domains: a FERM (ㄸ․1, Ezrin, Radixin, Moesin) binding domain, two WW domains, and a C-terminal SARAH domain. Salvador proteins localize to two distinct cortical regions. In flies, this cortical localization is mediated, in part, through association with the FERM domain-contain protein Merlin and the IgGdomain cell adhesion molecule Echinoid (Chishti et al., 1998; Yu et al., 2010; Yue et al., 2012; Su et al., 2017). Echinoid adhesive complexes form at the apical margin of adherens junctions, which are the most apical of the lateral junctions in insects (Fulford et al., 2018). Echinoid recruits Salvador to the cortex adjacent to adherens junctions (Yue et al., 2012; Sun et al., 2015). Alternatively, Merlin, in association with Kibra, recruits Salvador to the apical membrane (Yu et al., 2010; Su et al., 2017). Each of these membrane domains represent sites of Hippo pathway activation (Sun et al., 2015; Su et al., 2017).

A compelling model for the mechanism by which Salvador activates Hippo kinases is supported by structural and biochemical studies of fly and human Salvador (Bae et al., 2017; Cairns et al., 2018; reviewed in Bae and Luo, 2018). In this model, Salvador participates in hetero-tetrameric complexes consisting of two Salvador proteins and two Hippo/MST1/2 kinases (Figure 4B). Formation of this hetero-tetramer promotes kinase trans-autophosphorylation.

While formation of these structures may stabilize Hippo/MST1/2 kinase interactions, they are not essential for kinase activation per se. Purified MST1/2 kinases are fully active in vitro, suggesting they can self-activate independently of co-factors. In vivo, the requirement for Salvador may be bypassed in some contexts, when independent activating kinases are present. Consistent with a bypass mechanism, the GCK sub-family kinase, Tao-1, phosphorylates and activates Hippo in Drosophila eye and wing primordia. In these tissues, loss of Tao-1 leads to overgrowth phenotypes that are associated with decreased Hippo kinase activation (Boggiano et al., 2011; Poon et al., 2011). If the Salvador dependence can be bypassed, why is Salvador thought to be required for Hippo pathway activation?

One possibility is that Salvador has additional functions in activation of Hippo kinases, some of which may be contextdependent. Consistent with this interpretation, formation of fly Salvador-Hippo complexes blocks accessibility of the Hippo SARAH domain for binding to RASSF, a Hippo kinase inhibitor (Polesello et al., 2006; Ribeiro et al., 2010). However, evidence from mammalian cells supports both positive and negative regulation of Hippo kinase activity by mammalian RASSF family proteins (Avruch et al., 2009; Richter et al., 2009; Ni et al., 2013; Aruna et al., 2017). It may be that the differing results from the fly and mammalian systems are simply due to different pathway configurations between the cell types examined (see discussion in Rawat and Chernoff, 2015).

A second potential function for Salvador is to block an inactivating phosphatase. The STRIPAK complex antagonizes Hippo kinase activity by removal of activating phosphates. Human Salvador1 binds to, and inhibits, the catalytic subunit of PP2A of the STRIPAK complex in 293FT cells (Bae et al., 2017). A third possibility is that Salvador may be essential to enrich active Hippo at specific cellular locations, such as the apical or sub-apical membrane domains of epithelial cells (Yin et al., 2013; Su et al., 2017).

More work is needed to understand context-dependence for Salvador functions. Beyond epithelia, Hippo-like kinases function upstream of NDR kinases in other polarized cell types such as neurons, but the sub-cellular distribution of Salvador regulators, like Merlin, are less understood (Emoto et al., 2004, 2006; Reddy and Irvine, 2011). Broadening our exploration of these pathways in distinct tissue contexts will illuminate apparently conflicting data on Hippo regulation.

\section{Animal Furry Family Proteins Promote the Activation of Tricornered-Like Kinases}

Furry family proteins are essential components of Tricorneredlike NDR signaling systems in specific cellular contexts, from yeast to humans (Cong et al., 2001; Du and Novick, 2002; Hirata et al., 2002; Gallegos and Bargmann, 2004; Chiba et al., 2009; Norkett et al., 2019; Goto et al., 2010). Binding between Furry and Tricornered-like kinases is reported for fly and mammalian proteins (Chiba et al., 2009; Fang et al., 2010), but little is known about the structural features that make Furry proteins essential to Tricornered-like activity. Furry proteins are large, ranging 2000-3000 residues in length, and contain five to six conserved regions (Nagai and Mizuno, 2014). Of these, the $\mathrm{N}$-terminal-most region contains Armadillo repeats that may be a platform for protein-protein interactions with multiple partners (Tewari et al., 2010). However, aside from this domain, the function of the remaining conserved sequences is unknown (Nagai and Mizuno, 2014).

In HeLa cells, Furry is required for chromosomal alignment during metaphase (Chiba et al., 2009). Furry is required for activation of the Tricornered-like kinase, STK38, in both genetic and biochemical assays; and this function is enhanced by Mob2. Furry most-likely forms a complex with Mob2-STK38, based on co-immunoprecipitation. Notably, Furry-Mob2 binding is only detected under conditions of pathway hyperactivation, when cells are treated with a phosphatase inhibitor. Conversely, Mob2STK38 binding appears to be constitutive and insensitive to STK38 phosphorylation status. In dividing HeLa cells, STK38 kinase activity levels are cell-cycle dependent and reach their maximum at metaphase. At this stage, Furry co-localizes extensively with spindle-microtubules. Together, these findings raise the following critical questions: Does STK38 activation require the formation of a Furry-Mob2-STK38 ternary complex, or is STK38 activation a pre-condition for complex assembly? In either case, it is unclear how the hippo-like kinase, MST2, associates with these proposed complexes and whether Furry restricts STK38 function to the mitotic spindle. 


\section{DISTINCT MOBS FORM DEDICATED ACTIVATION COMPLEXES FOR THEIR NDR KINASE PARTNERS TO REGULATE DISTINCT PHYSIOLOGICAL EVENTS IN FUNGI}

Budding and fission yeast, as well as filamentous fungi, are each equipped with two classes of NDR family kinases. Each kinase is independently regulated by dedicated activation complexes, which consist of a Mob family protein, a STE20-like kinase, and a molecular scaffold. Following activation, each of the two classes of fungal NDR kinases, in complex with their exclusive Mob partners, participates in distinct cell biological roles. Generally, the fungal Warts/LATS kinases are key regulators of cell cycle progression while the Tricornered-like kinases play essential roles in cellular morphogenesis. A filamentous fungal species, the mold Neurospora crassa, has four Mobs, three of which interact with dedicated NDR kinase partners, and a fourth Mob3/Phocein, which does not (Maerz et al., 2009). In filamentous fungi, Mob3/Phocein functions in the hyphal growth phase and in sexual development (Maerz et al., 2009; Fu et al., 2011), as do other proteins in the fungal STRIPAK complex (Bloemendal et al., 2012). The striking parallels between fungal and animal STRIPAK-Hippo antagonism in regulation of actin cytoskeletal organization and formation of cellular protrusions such as filopodia or dendrites are reviewed by Kuck et al. (2019).

\section{Fungal Warts/LATS Kinases Play Key Roles in Cell Cycle Progression}

The prototypical NDR kinase, S. cerevisiae Dbf2, is a Warts/LATS-type kinase identified in a genetic screen for defective cell division, detected by the shape of connected daughter-cell pairs (Johnston and Thomas, 1982; Johnston et al., 1990). Subsequently, a paralogous Warts/LATS kinase, Dbf20, was identified (Toyn et al., 1991). Dbf2/20 are central components of the MEN, a signaling pathway that promotes disassembly of the mitotic spindle, chromosome de-condensation, and cytokinesis (Luca and Winey, 1998b; reviewed in Bardin and Amon, 2001; Hotz and Barral, 2014). Dbf2/20 activation is necessary to exit mitosis and relies on a dedicated multi-protein complex comprised of: Cdc15, a Ste20-like protein kinase; Mob1, the prototypical Mob; and Nud1, a scaffold protein. Mutation of any one of these genes results in mitotic exit failures.

In S. pombe, Sid2, also in the Warts/LATS NDR kinase group, functions in an analogous signaling pathway: The SIN (Hou et al., 2000; Salimova et al., 2000; Bardin and Amon, 2001). This signaling network promotes cytokinesis. Activation of Sid2, the terminal kinase in the SIN, relies on inputs from Sid1, a Ste20like protein kinase; Mob1, a Mob family protein, and Cdc11-Sid4 a scaffold complex. The $S$. pombe Cdc11 is the homolog of the S. cerevisiae Nud1 scaffold protein (Tomlin et al., 2002). Loss of function in sid2 or its co-activators results in the formation of multinucleated cells due to failed cytokinesis following DNA synthesis and mitosis (Krapp and Simanis, 2008). The core kinase cassettes of the MEN and SIN are largely conserved in flies and mammals, where they function as essential components of the Hippo pathway (Hergovich and Hemmings, 2012).

\section{Fungal Tricornered-Like Kinases Play Key Roles in the Regulation of Cellular Morphogenesis}

The first Tricornered-like NDR kinase, Cot-1, was identified in the filamentous fungus, Neurospora crassa (Yarden et al., 1992). In N. crassa, cot-1 mutants exhibit morphogenetic defects associated with impaired hyphal tip elongation and excessive branching. Homologous Tricornered-like NDR kinases were subsequently identified in S. cerevisiae and S. pombe: Cbk1 and Orb6, respectively (Fulvia et al., 1995, 1998; Nasr et al., 1996; Racki et al., 2000). Like Cot-1, Cbk1 and Orb6 have demonstrated roles in the regulation of cellular morphology. In S. cerevisiae, Cbk1 is a central kinase in the RAM pathway (Bidlingmaier et al., 2001; Nelson et al., 2003). Activation of Cbk1 requires inputs from Kic1, a Ste20-like protein kinase; Mob2, a Mob family protein, and Tao3/Pag1, a Furry like protein scaffold (reviewed in Weiss et al., 2002; Nelson et al., 2003). Loss of function in Cbk1 or its co-activators leads to defects in cell shape changes associated with mating, including a reduction in apical growth and impaired formation of polarized mating projections (Bidlingmaier et al., 2001). In S. pombe, Orb6 functions in the analogous MOR (Hachet et al., 2012). Activation of Orb6 requires inputs from Nak1, a STE20-like protein kinase; Mob2, a Mob family protein, Mor2, a Furry family scaffold protein. Orb6 loss of function or loss of other MOR network components results both in defective actin polarization and polarized cell growth.

\section{Fungal Mob1 and Mob2 Proteins Have Dedicated NDR Kinase Binding Partners}

In each of the model fungi, S. cerevisiae, S. pombe, and N. crassa, the two classes of NDR family kinases have dedicated Mob family binding partners (Maerz et al., 2009; Parker et al., 2019). This differs from Mob-NDR partnering dynamics in animals where there is genetic and biochemical evidence for Mob-NDR promiscuity. How is partner selectivity determined in these fungal systems?

The structural features that ensure specific Mob-NDR partner interactions in fungi are emerging (Aharoni-Kats et al., 2018). Studies of S. cerevisiae Mob-NDR complexes show that Mob1 and Mob2 each have a tri-peptide motif that confers binding selectivity, called kinase restrictor motifs (Parker et al., 2019). These motifs are within the NDR kinase binding-surface and have the sequence Arg-Gly-Glu in Mob1, and Lys-Tyr-Val in Mob2 (Parker et al., 2019). Indeed, exchange of this kinase restrictor motif between the two yeast Mobs is sufficient for the two chimeric Mobs to exchange their NDR kinase binding partners in a pull-down assay from cell lysates. Additionally, the chimeric Mobs bind to non-cognate NDR kinases with high affinity. Thus, the kinase restrictor motif determines the selectivity of Mob binding to a specific NDR kinase in S. cerevisiae.

Intriguingly, the $S$. cerevisiae Mob1 kinase restrictor motif is conserved among Class I Mob family proteins across a wide range 
of eukaryotes including invertebrates, vertebrates, and plants. Conversely, the $S$. cerevisiae Mob2 kinase restrictor motif is not conserved in animal Class II Mobs. Perhaps divergence from $S$. cerevisiae Mob1 kinase restrictor motif sequence underlies their differences in binding to Warts/LATS kinases versus Tricornered-like kinases.

\section{MOB-NDR KINASE SIGNALING IN Drosophila HIPPO AND HIPPO-LIKE PATHWAYS}

While the work carried out in fungi provides an indispensable foundation for understanding Class I and Class II Mobs, the expansion of the animal mob gene family in multicellular organisms is associated with added complexity in the functions of Class I and Class II Mobs. In fungi, exclusivity in Mob-NDR kinase partnering allows for independent regulation of distinct NDR kinases with distinct functions in fungal physiology. In contrast, some animal Mobs exhibit promiscuity with respect to their NDR binding partners. The molecular and cell-biological implications for NDR co-regulation by shared Mob binding partners are only beginning to be appreciated (see Gundogdu and Hergovich, 2019). How this impacts cell and tissue function will depend on the combination of Mobs and alternative NDR kinase co-regulators within that cell type. To highlight the functional outputs that are controlled by Mob-dependent pathways, we focus on physiological and morphological studies in Drosophila, where genetic methods have facilitated in vivo analyses.

\section{An Expanded Set of Co-activator Proteins Regulate the Activation of Drosophila NDR Kinases}

The fly genome encodes a single kinase from each NDR kinase subfamily: Warts and Tricornered. However, there is one representative from each of the four animal Mob classes. The four fly genes encoding Mob-family proteins are named mats, mob2, mob3, and mob4 (Ye et al., 2009). As for the mammalian Mobs, the Class I fly Mob, Mats, binds to both Warts and Tricornered kinases. In contrast, Mob2 has only been reported to bind to Tricornered kinase (He et al., 2005b). Consistent with the observed physical interactions, Mats functions in fly Hippo signaling as an essential Warts co-activator. Tests for genetic interactions with tricornered support a role for Mats as a Tricornered kinase co-activator (Geng et al., 2000; He et al., 2005b). Conversely, the cell biological role of Mob2 is unclear.

Flies have an expanded set of GCK subfamily kinases, relative to fungi, with nine genes. Of these, hippo, happyhour, misshapen, and gckIII are known to function upstream of fly NDR kinases (Staley and Irvine, 2012; Li et al., 2014; Zheng et al., 2015; Poon et al., 2018). Lastly, the Drosophila genome encodes two scaffold proteins that are required for NDR kinase activation: salvador and furry (Cong et al., 2001; Kango-Singh et al., 2002; Tapon et al., 2002). The following sections discuss the fly pathways that involve these genes, and the physiological processes that the pathways control.

\section{Fly Warts NDR Kinase Is Central to the Highly Conserved Hippo Growth-Restrictive Pathway}

Since the seminal identification of the Hippo pathway, multiple upstream regulators and downstream targets have been identified that implicate the core Hippo signaling pathway, comprised of Hippo kinase, Warts kinase, Mats (Mob1), and Salvador, as a key regulator for a diverse set of cellular and physiological functions, including stem cell maintenance, cellular differentiation, epithelial cell mechano-transduction, cytoskeletal dynamics, cell migration, organogenesis, tissue homeostasis, and pathology (reviewed in Staley and Irvine, 2012; Matsui and Lai, 2013; $\mathrm{Yu}$ and Guan, 2013; Irvine and Harvey, 2015; Fallahi et al., 2016; Chen et al., 2019; Sahu and Mondal, 2019; Snigdha et al., 2019). In this section, we focus only on the specific studies that identified the genes and illuminated our understanding of the signaling pathways.

Many components of the animal Hippo signaling pathway were identified through forward genetic screens in flies (reviewed in Kim and Jho, 2018b; Gokhale and Pfleger, 2019). In 1993, Bryant et al. (1993) sought to identify tumor suppressor genes using a genetic mosaic screen, which identified warts based on the "spectacular outgrowths from the body surface" that formed from warts mutant epithelial cells. Then in 1995, two groups independently cloned the warts gene, one group naming the gene lats for its identified tumor suppressor phenotype (Justice et al., 1995; Xu et al., 1995), leading to identification of warts as a Dbf2-related kinase. Since then, many groups used genetic mosaic screens to identify growth suppressors through recovery of mutant alleles that produced epithelial tissue overgrowth phenotypes. The first Hippo signaling pathway gene identified through such screens was salvador, independently isolated by two groups (Kango-Singh et al., 2002; Tapon et al., 2002). Overgrowth of salvador mutant epithelial cells arises from both increased cell proliferation and reduced apoptotic cell death.

The hippo gene, named due to the enlarged heads that result from presence of hippo loss of function cells in mosaic developing head epithelia, was identified by five groups (Harvey et al., 2003; Jia et al., 2003; Pantalacci et al., 2003; Udan et al., 2003; Wu et al., 2003). Cells that lack Hippo kinase, similar to those that lack Warts kinase or the Salvador scaffold, show increased cellular proliferation with reduced levels of the Drosophila inhibitor of apoptosis, DIAP1, and increased levels of the G1-S cell cycle regulator, Cyclin E (Kango-Singh et al., 2002; Tapon et al., 2002). Genetic experiments placed these genes in the same growth restricting, or tumor suppressor pathway. Furthermore, Hippo, Warts, and Salvador proteins physically bind to each other in biochemical assays. Altogether, these data indicated that Hippo, Warts, and Salvador are essential components of a STE20 kinaseNDR kinase signaling pathway.

The Mob component of the Hippo pathway was discovered by Lai et al. (2005), who identified the Class I Mob, Mats, as an essential co-activator of Warts kinase. Like other fly Hippo pathway genes, mats mutant cells in mosaic developing tissues lead to enlarged adult tissues. Together, these studies 
established the fly Hippo-Warts-Mats-Salvador intracellular signaling pathway, which restricts tissue growth.

Shortly after the discovery of Mats, the transcription factor Yorkie (named for homology to the mammalian Yes associated protein or YAP) was identified as a critical target of Warts kinase (Huang et al., 2005). When a substantial proportion of developing adult structures consist of cells that lack yorkie function, a miniaturized fly forms, with otherwise normal morphologies and viability. Conversely, Yorkie overexpression leads to striking tissue overgrowth. These are the opposite phenotypes obtained for similar experiments with Warts kinase, or its activators, where large numbers of cells lacking the upstream pathway genes give rise to overgrown adult tissues. Genetic experiments placed yorkie downstream of warts and biochemical assays demonstrated that Yorkie is a Warts kinase phosphorylation target (Huang et al., 2005). It was later shown that Warts-dependent phosphorylation of Yorkie generates a binding site for 14-3-3 proteins (Dong et al., 2007). Phosphorylated Yorkie is therefore sequestered in the cytoplasm, which prevents its transcriptional regulatory activity (Dong et al., 2007; Oh and Irvine, 2008; Zhang et al., 2008; Oh and Irvine, 2009; Ren et al., 2010).

Several studies suggest a non-transcriptional role for Yorkie. In epithelial cells of the developing wing and eye, and in follicular epithelia of adult ovaries, a small fraction of the total cellular Yorkie is at the apical cortex (Oh et al., 2009; Fletcher et al., 2018; Xu et al., 2018). This apical Yorkie pool promotes activation of non-muscle myosin II in developing wing epithelial cells, independently of Yorkie's nuclear role as a DNA-binding transcriptional regulator (Xu et al., 2018). Furthermore, cells mutant for warts show strong enrichment of cortical Yorkie. Although this finding suggests that Warts kinase limits Yorkie apical localization, it does not address whether the Warts kinase needs to be fully activated by a combination of Mob and STE20 kinase actions. In this regard, it will be illuminating to determine whether Mats is required for Yorkie apical localization.

\section{Fly Tricornered Kinases Acts in a Hippo-Like Pathway Controlling Cellular Morphogenesis}

Tricornered is emerging as a genetic regulator of cellular morphogenesis in several fly tissues and its regulatory partners are coming to light. Overall, studies of the fly Tricornered kinase have lagged behind those of the fly Warts/LATS kinase. The tricornered gene was identified in 1976; tricornered mutant cells produce split and morphologically aberrant wing hairs (Ferrus, 1976; Gubb and García-Bellido, 1982; Vinson and Adler, 1987). The Adler lab determined that tricornered encodes a NDR kinase (Geng et al., 2000). The furry gene was later identified in a genetic screen for wing hair polarity mutants and showed a genetic interaction with tricornered (Cong et al., 2001). Wing epithelial cells that are mutant for either tricornered or furry, produce multiple, split, and aberrantly shaped wing hairs, a phenotype associated with defects in actin bundling (Geng et al., 2000; Cong et al., 2001; He et al., 2005b; Fang and Adler, 2010).

Fly Tricornered regulation by Mob proteins is poorly understood. Potential genetic interactions between tricornered and each of the four fly mob genes have been probed by assessing their effects on wing hair formation. Forced expression of a non-activatable tricornered ${ }^{T 453 A}$ transgene in wing epithelial cells produces multiple hairs (He et al., 2005b; Fang and Adler, 2010). The T453A mutation abolishes the HM threonine that is phosphorylated by Hippo and GckIII (He et al., 2005b; Emoto et al., 2006; Poon et al., 2018; see also Millward et al., 1999). When tricornered ${ }^{T 453 A}$ overexpressing wing epithelial cells are also hemizygous for any one of the four mob genes, they more frequently produce aberrant wing hairs, and the defects are more extreme. These data implicate multiple Mob family proteins in Tricornered NDR kinase pathways. However, to date, Mats and Mob2 are the only fly Mobs demonstrated to physically bind Tricornered (He et al., 2005a), and the links between these observations and the underlying cell biology are not clear. Subsequently, studies in the pupal eye imaginal disc implicated Mob2 as a regulator of photoreceptor cell morphogenesis (Liu et al., 2009). In this tissue, RNAi mediated knock-down of mob2 results in defective rhabdomere formation and pigment cell differentiation. Although these studies implicate Mob2 function in cellular morphogenesis, the underlying mechanism is unclear, because Tricornered function was not investigated in this tissue.

Additional genetic evidence that fly Mob2 is required for Tricornered activation comes from studies of the larval neuromuscular junctions, where synaptic contacts form between motor neuron axons and muscle. Campbell and Ganetzky (2013) showed that mob2 is required for normal development of neuromuscular junctions. Each junction involves multiple neuronal contacts called synaptic boutons. Neurons with reduced mob2 function produce broad axonal extensions with more synaptic boutons relative to controls. The presence of a heterozygous mutation in tricornered worsened the mob2 junction defects. Presence of a heterozygous mutation in warts however gave no alteration. Subsequently, RNAi depletion of tricornered in neurons gave defects resembling the mob2 synaptic bouton defects (Natarajan et al., 2015). These investigators detected reduced presynaptic levels of the Actin regulator, WASP (Wiskott- $\underline{\text { Aldrich }}$ Syndrome $\underline{\text { Protein) in tricornered }}$ mutant larvae, accompanied by reduced wasp mRNA. This latter result raises the question of whether Tricornered may regulate downstream transcription factors but provides no evidence for whether such regulation may be direct or indirect. Taken altogether, the data from studies of neurons in flies support a role for Tricornered in regulation of the actin cytoskeleton and roles for both Tricornered and Mob2 in formation of synaptic boutons. Parallels have been observed in mammalian neurons, where NDR kinases phosphorylate proteins involved in endocytosis and vesicle trafficking, substrates required for dendrite growth (Ultanir et al., 2012; Leger et al., 2018).

Tricornered and Furry also have related functions in the regulation of larval neuronal morphogenesis (Emoto et al., 2004, 2006). Drosophila sensory neurons of the larval ectoderm form large arbors of dendrites. Tricornered function is required for both normal dendrite morphogenesis and the tiled organization of dendritic branches, where the dendritic arbors from different sensory neurons are organized in non-overlapping patterns 
(Emoto et al., 2004, 2006; reviewed in Jan and Jan, 2010; Parrish, 2016). Neurons mutant for either tricornered or furry have excessive and overlapping dendritic branches (Emoto et al., 2004, 2006; Koike-Kumagai et al., 2009). Subsequently, Norkett et al. (2019) have shown that Tricornered, in partnership with Furry, regulates neurite growth through phosphorylation of the kinesin-like protein Pavarotti, in a mechanism that inhibits microtubule sliding (Del Castillo et al., 2015; Norkett et al., 2019). It will be exciting to see whether Mob proteins participate in this process, and if so, which classes of Mobs are involved.

Hippo kinase functions biochemically and genetically upstream of both Tricornered and Warts kinases to regulate sensory neuron dendritic tiling and maintenance, respectively (Emoto et al., 2006). Overexpression of transgenic wild-type tricornered rescues tiling defects of hippo mutant neurons. This result supports a role for Tricornered downstream of Hippo kinase but raises the question of whether another STE20 kinase can bypass the requirement for Hippo kinase to fully activate Tricornered. Alternatively, elevated levels of partially activated Tricornered, perhaps with a Mob partner, might be sufficient to mediate repulsive signaling that blocks dendrites from extending into the vicinity of a different neuron. To understand context-dependent Hippo and Hippo-like pathway activities, it will be important to explore the relationships between pathway components in this peripheral nervous system context. If Hippo is the activating kinase for both Tricornered and Warts in sensory neurons, the regulation of NDR kinase switching, as well the potential for NDR kinase competition for Hippo kinase, could be investigated productively with the same assays.

Results from other organs implicate additional STE20 kinases in genetic Tricornered pathways. Both tricornered and furry regulate egg elongation during oogenesis (Horne-Badovinac et al., 2012). When follicular epithelial cells are mutant for either gene, they form round eggs instead of the normal ellipsoid eggs. Follicular epithelial cells require Tricornered for anisotropic egg elongation into an ellipsoid shape, whereas Hippo appears to be dispensable (Meignin et al., 2007; Polesello and Tapon, 2007; Yu et al., 2008; Yan et al., 2011). Instead, a MAP4K kinase, Misshapen, is required. When most follicular epithelial cells are mutant for misshapen, the resultant round eggs resemble those formed by tricornered or furry mutant epithelial cells (HorneBadovinac et al., 2012; Lewellyn et al., 2013). Thus, Misshapen is part of an egg elongation regulatory system; as such, it is a candidate activating kinase for Tricornered in this system. However, this potential pathway relationship has not been tested, either in vivo by genetics or in vitro in cultured cells (reviewed in Gates, 2012; Cetera and Horne-Badovinac, 2015).

A third STE20 kinase, GckIII, is a bonafide activator of Tricornered kinase in the morphogenesis of larval tracheae (Figure 1; Song et al., 2013; Poon et al., 2018). However, Mob function in tracheal morphogenesis was not addressed. These results highlight the value of proteomic and forward genetic screens for uncovering new contexts where alternative NDR kinase activation pathways should be investigated critically. Such studies will be needed to assess whether Mobs are required for full NDR kinase activation when the NDR kinase is activated by a STE20 kinase that differs from Hippo.

\section{Emerging Roles for the Class IV Mobs as Components of the Highly Conserved STRIPAK Complex}

In this section we discuss emerging roles for the most divergent class of Mob family proteins - the Phocein or Class-IV Mobs. In flies and mammals, Mob4/Phocein proteins have been identified as components of the highly conserved STRIPAK complex. Filamentous fungi have a Phocein-like Mob, which shares developmental phenotypes with genes encoding STRIPAK components, which suggests that Class IV/Phocein proteins have conserved function across multicellular eukaryotes (Maerz et al., 2009; Fu et al., 2011; Bloemendal et al., 2012).

As a component of STRIPAK, Mob4/Phocein is a negative regulator of fly Hippo signaling. It remains unclear whether the Mob4/Phocein proteins act exclusively via STRIPAK or whether, like Class I and Class II Mobs, they can also function as direct regulators of NDR family kinases. The two classes of NDR family kinases share many of their regulators, it will be challenging to unravel the mechanisms that ensure balanced signaling through each of the two kinases is adequately coordinated for their functions in any specific cell type. Pursuing the role of Mob4/Phocein function in relation to that of STRIPAK is a compelling entry into this question, because of its potential to restrict NDR kinase signaling via the regulation STE20 kinases (e.g., Hippo, Misshapen, GckIII, and their mammalian homologs). Consistent with this notion, multiple mammalian STE20 kinases of the GCK II and III subfamilies interact with STRIPAK components (Sugden et al., 2013; Hwang and Pallas, 2014).

\section{The STRIPAK Complex Is a Negative Regulator of Hippo Signaling}

Phosphorylation of NDR kinases is required for their full activation. In vivo, this activation is dampened by protein phosphatases (Hergovich et al., 2006b). The first evidence that PP2A decreases NDR kinase activity (Millward et al., 1999) took advantage of a potent phosphatase inhibitor, okadaic acid, which preferentially inactivates PP2A (Takai et al., 1995; Favre et al., 1997). Both increased kinase activity and increased phosphorylation of the human Tricornered-like kinase were observed following treatment of COS-1 cells with okadaic acid (Millward et al., 1999). Subsequent studies of yeast and fly NDR kinases reported similar effects in response to okadaic acid treatment, indicating that negative regulation of NDR kinases by phosphatase activity is conserved from yeast to humans (Mah et al., 2001; Chan et al., 2005; Lai et al., 2005; Hergovich et al., 2006a; Koike-Kumagai et al., 2009; Zheng et al., 2017).

Proteomic studies linked PP2A regulation of Hippo signaling pathways to STRIPAK, a highly conserved multimeric protein complex that includes PP2A, a Mob/Phocein protein, and STE20 kinases of the GCK subfamily (Figure 4B; Glatter et al., 2009; Goudreault et al., 2009; Hyodo et al., 2012; Sugden et al., 2013; Madsen et al., 2015; Shi et al., 2016). Moreover, studies in flies and mammals have shown that STRIPAK is a potent negative regulator of Hippo signaling (Ribeiro et al., 2010; Couzens et al., 2013; Zheng et al., 2017; Gil-Ranedo et al., 2019; Tang et al., 2019). 
In this role, PP2A in the STRIPAK deactivates Hippo kinases, which in turn leads to decreased Warts/LATS kinase activation. Notably, fly Mob4 and the STRIPAK complex are required for normal formation of synaptic boutons at fly neuromuscular junctions, a process regulated by Tricornered and Mob2 (Schulte et al., 2010; Neisch et al., 2017).

Our current understanding of the structural basis for STRIPAK complex assembly is limited, but exciting new details are emerging (Tang et al., 2019). Tang and colleagues examined interactions between major mammalian STRIPAK components and propose a "two-arm" model of STE20 kinase recruitment to the STRIPAK complex. In this model, the proteins STRIP1 and SLMAP (Strip and Slmap in flies), bind MST2 and MST4 kinases (Hippo and GckIII in flies) at distinct interaction sites (diagrammed in Figure 5B). Consistent with STRIPAK's role as a negative regulator, these binding interactions are phosphorylation dependent, indicating that only activated STE20 kinases are recruited to the STRIPAK complex.

The roles of other STRIPAK components are less understood. An intact STRIPAK complex is required for negative regulation of Hippo signaling in distinct Drosophila cellular contexts, but the contribution of Mob4/Phocein has yet to be defined (Ribeiro et al., 2010; Schulte et al., 2010; Sakuma et al., 2016; Zheng et al., 2017; Neisch et al., 2017; Gil-Ranedo et al., 2019). Mob4/Phocein proteins have been shown to bind directly to STE20 kinases in both fly and mammalian cells (Ribeiro et al., 2010; Couzens et al., 2017; Chen et al., 2018), but it is unclear if Mob4/Phocein is required to recruit GCK-type STE20 kinases to the STRIPAK complex. To function as a recruiting protein, Mob4/Phocein must function as an adaptor between its STE20 kinase binding partner and one or more STRIPAK components. However, the nature of the Mob-STRIPAK binding interaction is unknown, even though the founding member of the Class IV/Phocein Mobs was identified as a rat Striatinbinder using yeast two-hybrid screens (Baillat et al., 2001; see also Gordon et al., 2011). Alternatively, Mob4/Phocein proteins may contribute to STE20 kinase silencing through competitive interactions with Class I Mobs (Figure 5C). By blocking Class I Mob access to activating STE20 kinases, Mob4/Phocein proteins would prevent formation of a Warts/LATS ternary activation complex (Figure 5A). Many open questions remain about Mob participation in STRIPAK complexes and their contributions to STRIPAK-dependent negative-regulation of Hippo-like pathways.

\section{CONCLUDING REMARKS}

Most animal Mob studies have focused on their roles as NDR kinase activators, now well-established by combinations of genetic, cell biological, and biochemical approaches. Potential roles independent of Hippo and Hippo-like pathways exist, and remain to be explored further (Hergovich et al., 2006b; Hergovich, 2016). Our expanded understanding of animal Mobs has largely come from the research enterprise investigating the Hippo growth regulation pathway. However, as new cellular contexts for Hippo signaling or NDR kinase activation are uncovered, the question of whether Mobs are involved is increasingly overlooked. The role of Mobs in full activation of Warts/LATS and Tricornered-like kinases would provide a tool to explore whether alternative STE20 kinases result in weaker activation of the downstream NDR kinases.

Even the roles of Mobs as NDR kinase activators need to be clarified. Class I Mobs are demonstrated co-activators of Warts/LATS NDR kinases in flies, mice, and humans, with defined binding interactions. However, Class II Mob functions in NDR kinase regulation remain unclear. Fly and mammalian Class II Mobs have been shown to bind exclusively to Tricorneredlike NDR kinases, but currently there is no consensus concerning the nature of these interactions. Some studies suggest that Class II Mobs promote activation of Tricornered-like kinases, others suggest an inhibitory function. Furthermore, the roles of Class I and Class II Mobs are increasingly overlooked in studies of both Hippo kinase signaling and inputs into the Hippo signaling pathway from alternative GckIII and MAP4K kinases.

Localization of Hippo signaling components to distinct membrane domains is a recurring theme in polarized cell types (Fulford et al., 2018). In at least one case, localization is coincident with competitive binding interactions that prevent binding of the Mob1 activator for Warts/LATS kinase (Dutta et al., 2018). However, other studies that investigate differential localization of activated Warts/LATS kinase do not test for involvement of a Mob partner. Another study suggests that Mob activation is not essential as the first step for Warts/LATS activation, but that it is necessary to fully activate the kinase (Mana-Capelli and McCollum, 2018). Whether activation by a Mob can be bypassed remains an open question that becomes more important, as more alternative pathways to activate Warts/LATS and Tricorneredlike kinases are uncovered.

Early studies of Class 1 Mobs in yeast and flies demonstrated an essential role in activation of their partner NDR kinases. While current models suggest a more flexible requirement, this activity raises the question of whether the availability of Mob adaptors is regulated within cells, either through transcriptional or posttranscriptional mechanisms, and whether Mob protein activity is regulated independently from their partners. These questions about regulation of Mob levels have been best studied in mouse and human systems (Sasaki et al., 2007; Lignitto et al., 2013; Otsubo et al., 2017; Chen et al., 2018; Kim and Jho, 2018a), as covered recently in Gundogdu and Hergovich (2019).

Phylogenetic comparisons indicate that Mobs exhibit functional diversity, paralleled by sequence diversity of animal Mob classes. Multiple experimental approaches suggest that different animal Mob classes have partially overlapping, yet distinct binding partners, many of which are conserved across eukaryotes. This review focused on those conserved core functions. Class I Mobs are phosphorylated by Hippo-like kinases, but are they phosphorylated by other STE20 kinases? Are Class II Mobs also phosphorylated by Hippo kinases or others? Class II Mobs bind exclusively to Tricornered-like NDR kinases, but we lack a consensus view of the functional consequences for these interactions. Does the lack of consensus reflect a context-dependent difference, or differences in the types of assays used? 
Proteomic studies of mammalian Class III and IV/Phocein Mobs suggest that these proteins do not bind NDR kinases. In contrast, Class IV/Phocein Mobs are essential components of the STRIPAK complex that antagonizes Hippo signaling in animals, a role also implicated by genetic studies of filamentous fungi (Shomin-Levi and Yarden, 2017; Kuck et al., 2019). In spite of this conserved requirement across model organisms, we know little about the molecular aspects of Mob4/Phocein function within STRIPAK. Mob4/Phocein proteins stand out as a potential regulatory node, through these Mobs' ability to associate with both STRIPAK-associated phosphatase and GCKtype STE20 kinases. What is the nature of the STE20 kinase association with STRIPAK, is it purely a phosphatase substrate or does it have a different role? The role of Class IV/Phocein Mobs in recruiting substrates to STRIPAK for dephosphorylation is an open question.

Identification of Class IV/Phocein Mobs and STRIPAK complex components in filamentous fungi raises questions about the apparently divergent regulation of Hippo-related STE20 kinases in unicellular yeasts, $S$. cerevisiae and Schizosaccharomyces pombe. These yeasts have a homolog of phosphatase PP2A, but not of other protein components of the STRIPAK complex, such as Striatin. Kuck et al. (2019) point to alternative yeast proteins that may substitute as PP2A-STE20 kinase linkers instead. These distinctions raise questions about divergence between unicellular and multicellular eukaryotes, and whether switching between upstream kinases to activate NDR kinases may be particularly important in organizing multicellular structures, such as the fruiting bodies of filamentous fungi (Beier et al., 2016). In this regard, it is striking that the ciliate Tetrahymena appears to have a Mob4 homolog (Soares et al., 2019). To understand the broadly conserved network of Mob functions in Hippo and Hippo-like pathways, it will be valuable to investigate whether ciliates, such as Paramecium and Tetrahymena, have STRIPAK complexes that regulate switching between the ciliate NDR kinase pathways.

\section{REFERENCES}

Aharoni-Kats, L., Zelinger, E., Chen, S., and Yarden, O. (2018). Altering Neurospora crassa MOB2A exposes its functions in development and affects its interaction with the NDR kinase COT1. Mol. Microbiol. 108, 641-660. doi: $10.1111 / \mathrm{mmi} .13954$

Arencibia, J., Pastor-Flores, D., Bauer, A., Schulze, J., and Biondi, R. M. (2013). AGC protein kinases: from structural mechanism of regulation to allosteric drug development for the treatment of human diseases. Biochim. Biophys Acta 1834, 302-321. doi: 10.1016/j.bbapap.2013.03.010

Aruna, B., Srinivas, S., Jessy, M., Harshada, M., and Ruchi, A. (2017). Rassf proteins as modulators of Mst1 kinase activity. Sci. Rep. 7:45020. doi: 10.1038/srep45020

Avruch, J., Xavier, R., Bardeesy, N., Zhang, X.-F., Praskova, M., Zhou, D., et al. (2009). Rassf family of tumor suppressor polypeptides. J. Biol. Chem. 284, 11001-11005. doi: 10.1074/jbc.R800073200

Bae, S. J., and Luo, X. (2018). Activation mechanisms of the Hippo kinase signaling cascade. Biosci. Rep. 38:BSR20171469. doi: 10.1042/BSR20171469

Bae, S. J., Ni, L., Osinski, A., Tomchick, D., Brautigam, C., and Luo, X. L. (2017). SAV1 promotes Hippo kinase activation through antagonizing the PP2A phosphatase STRIPAK. eLife 6:e30278. doi: 10.7554/eLife.30278.001

Baillat, G., Moqrich, A., Castets, F., Baude, A., Bailly, Y., Benmerah, A., et al. (2001). Molecular cloning and characterization of phocein, a protein found
We have emphasized the core functions of Mobs that are conserved across eukaryotes in this review; others have focused on the diverse roles of the numerous mammalian Mobs in distinct pathologies (Gundogdu and Hergovich, 2019). Additional functions for NDR kinases and Mobs are just beginning to show up from unbiased screens in distinct cell types, whether based on traditional genetics, RNAi, chemical genetics or protein-protein interactions. The potential for crossregulation and/or competition between Warts/LATS kinases and Tricornered-like kinases is apparent through their regulation by shared Class I Mob and Hippo activators but is only beginning to be investigated in detail (Zhang et al., 2018). Our understanding of both Mobs and Hippo pathway signaling would benefit from experiments to directly test whether a Mob-NDR kinase partnership and STRIPAK antagonism influence the pathway output in each new cellular context.

\section{AUTHOR CONTRIBUTIONS}

This manuscript is adapted from the dissertation authored by JD, in partial completion of his Ph.D. degree. LR contributed to writing of this manuscript.

\section{FUNDING}

The Raftery Lab was supported by the National Science Foundation (NSF) research grant 1355091 (LR).

\section{ACKNOWLEDGMENTS}

We thank the reviewers for insightful comments that improved this work and apologize to those whose work was not included due to space limitations or was otherwise overlooked.

from the Golgi complex to dendritic spines. Mol. Biol. Cell 12, 663-673. doi: $10.1091 / \mathrm{mbc} .12 .3 .663$

Bardin, A. J., and Amon, A. (2001). Men and sin: what's the difference? Nature reviews. Mol. Cell Biol. 2, 815-826. doi: 10.1038/35099020

Beier, A., Teichert, I., Krisp, C., Wolters, D. A., and Kück, U. (2016). Catalytic subunit 1 of protein phosphatase $2 \mathrm{~A}$ is a subunit of the STRIPAK complex and governs fungal sexual development. MBio 7:e00870-16. doi: 10.1128/mBio. 00870- 16

Bichsel, S. J., Tamaskovic, R., Stegert, M. R., and Hemmings, B. A. (2004). Mechanism of Activation of NDR (Nuclear Dbf2-related) Protein Kinase by the hMOB1 Protein. J. Biol. Chem. 279, 35228-35235. doi: 10.1074/jbc. M404542200

Bidlingmaier, S., Weiss, E. L., Seidel, C., Drubin, D. G., and Snyder, M. (2001). The Cbklp pathway Is important for polarized cell growth and cell separation in Saccharomyces cerevisiae. Mol. Cell. Biol. 21:2449. doi: 10.1128/MCB.21.7.24492462.2001

Bloemendal, S., Bernhards, Y., Bartho, K., Dettmann, A., Voigt, O., Teichert, I., et al. (2012). A homologue of the human STRIPAK complex controls sexual development in fungi. Mol. Microbiol. 84, 310-323. doi: 10.1111/j.1365-2958. 2012.08024.x

Boggiano, J. C., Vanderzalm, P. J., and Fehon, R. G. (2011). Tao-1 Phosphorylates Hippo/MST Kinases to Regulate the Hippo-Salvador-Warts tumor 
suppressor pathway. Dev. Cell 21, 888-895. doi: 10.1016/j.devcel.2011. 08.028

Bryant, P., Watson, K., Justice, R. W., and Woods, D. (1993). Tumor-suppressor genese encoding proteins required for cell-interactions and signal-transduction in Drosophila. Dev. Supp. 119, 239-249.

Cairns, L., Tran, T., Fowl, B. H., Patterson, A., Kim, Y. J., Bothner, B., et al. (2018). Salvador has an extended SARAH domain that mediates binding to Hippo kinase. J. Biol. Chem. 293:5532. doi: 10.1074/jbc.RA117.000923

Campbell, M., and Ganetzky, B. (2013). Identification of Mob2, a novel regulator of larval neuromuscular junction morphology, in natural populations of Drosophila melanogaster. Genetics 195:915. doi: 10.1534/genetics.113.156562

Cetera, M., and Horne-Badovinac, S. (2015). Round and round gets you somewhere: collective cell migration and planar polarity in elongating Drosophila egg chambers. Curr. Opin. Genet. Dev. 32, 10-15. doi: 10.1016/j.gde. 2015.01.003

Chan, E. H., Nousiainen, M., Chalamalasetty, R. B., Schafer, A., Nigg, E. A., and Sillje, H. H. (2005). The Ste20-like kinase Mst2 activates the human large tumor suppressor kinase Lats1. Oncogene 24, 2076-2086. doi: 10.1038/sj.onc.1208445

Chen, M., Zhang, H., Shi, Z., Li, Y., Zhang, X., Gao, Z., et al. (2018). The MST4-MOB4 complex disrupts the MST1-MOB1 complex in the Hippo-YAP pathway and plays a pro-oncogenic role in pancreatic cancer. J. Biol. Chem. 293, 14455-14469. doi: 10.1074/jbc.RA118.003279

Chen, Y. A., Lu, C. Y., Cheng, T. Y., Pan, S. H., Chen, H. F., and Chang, N. S. (2019). WW domain-containing proteins YAP and TAZ in the hippo pathway as key regulators in stemness maintenance, tissue homeostasis, and tumorigenesis. Front. Oncol. 9:60. doi: 10.3389/fonc.2019.00060

Chiba, S., Ikeda, M., Katsunuma, K., Ohashi, K., and Mizuno, K. (2009). MST2- and Furry-mediated activation of NDR1 kinase is critical for precise alignment of mitotic chromosomes. Curr. Biol. 19, 675-681. doi: 10.1016/j.cub.2009.02.054

Chishti, A. H., Kim, A. C., Marfatia, S. M., Lutchman, M., Hanspal, M., Jindal, H., et al. (1998). The FERM domain: a unique module involved in the linkage of cytoplasmic proteins to the membrane. Trends Biochem. Sci. 23, 281-282. doi: 10.1016/S0968-0004(98)01237-7

Chung, H.-Y., Gu, M., Buehler, E., Macdonald, M. R., and Rice, C. M. (2014). Seed sequence-matched controls reveal limitations of small interfering RNA knockdown in functional and structural studies of hepatitis C virus NS5AMOBKL1B interaction. J. Virol. 88:11022. doi: 10.1128/JVI.01582-14

Colman-Lerner, A., Chin, T. E., and Brent, R. (2001). Yeast Cbk1 and Mob2 activate daughter-specific genetic programs to induce asymmetric cell fates. Cell 107, 739-750. doi: 10.1016/S0092-8674(01)00596-7

Cong, J., Geng, W., He, B., Liu, J., Charlton, J., and Adler, P. N. (2001). The furry gene of Drosophila is important for maintaining the integrity of cellular extensions during morphogenesis. Development 128, 2793-2802.

Couzens, A. L., Knight, J. D., Kean, M. J., Teo, G., Weiss, A., Dunham, W. H., et al. (2013). Protein interaction network of the mammalian Hippo pathway reveals mechanisms of kinase-phosphatase interactions. Sci. Signal. 6:rs15. doi: 10.1126/scisignal.2004712

Couzens, A. L., Xiong, S., Knight, J. D. R., Mao, D. Y., Guettler, S., Picaud, S., et al. (2017). MOB1 mediated phospho-recognition in the core mammalian hippo pathway. Mol. Cell. Proteomics 16:1098. doi: 10.1074/mcp.M116.065490

Cui, X., Guo, Z., Song, L., Wang, Y., and Cheng, Y. (2016). NCP1/AtMOB1A plays key roles in auxin-mediated arabidopsis development. PLoS Genet. 12:e1005923. doi: 10.1371/journal.pgen.1005923

Dan, I., Watanabe, N. M., and Kusumi, A. (2001). The Ste20 group kinases as regulators of MAP kinase cascades. Trends Cell Biol. 11, 220-230. doi: 10.1016/ S0962-8924(01)01980-8

Das Thakur, M., Feng, Y., Jagannathan, R., Seppa, M. J., Skeath, J. B., and Longmore, G. D. (2010). Ajuba LIM proteins are negative regulators of the Hippo signaling pathway. Curr Biol. 20, 657-662. doi: 10.1016/j.cub.2010. 02.035

Davis, J. R., and Tapon, N. (2019). Hippo signalling during development. Development 46:dev167106. doi: 10.1242/dev.167106

Del Castillo, U., Lu, W., Winding, M., Lakonishok, M., and Gelfand, V. I. (2015). Pavarotti/MKLP1 regulates microtubule sliding and neurite outgrowth in Drosophila neurons. Curr. Biol. 25, 200-205. doi: 10.1016/j.cub.2014.11.008

Devroe, E., Erdjument-Bromage, H., Tempst, P., and Silver, P. A. (2004). Human mob proteins regulate the NDR1 and NDR2 serine-threonine kinases. J. Biol. Chem. 279, 24444-24451. doi: 10.1074/jbc.M401999200
Dewey, E. B., Sanchez, D., and Johnston, C. A. (2015). Warts phosphorylates mud to promote pins-mediated mitotic spindle orientation in Drosophila, independent of Yorkie. Curr. Biol. 25, 2751-2762. doi: 10.1016/j.cub.2015. 09.025

Dong, J., Feldmann, G., Huang, J., Wu, S., Zhang, N., Comerford, S. A., et al. (2007). Elucidation of a universal size-control mechanism in Drosophila and mammals. Cell 130, 1120-1133. doi: 10.1016/j.cell.2007.07.019

Du, L.-L., and Novick, P. (2002). Paglp, a novel protein associated with protein kinase Cbklp, is required for cell morphogenesis and proliferation in Saccharomyces cerevisiae. Mol. Biol. Cell 13, 503-514. doi: 10.1091/mbc.01-070365

Dutta, S., Mana-Capelli, S., Paramasivam, M., Dasgupta, I., Cirka, H., Billiar, K., et al. (2018). TRIP6 inhibits Hippo signaling in response to tension at adherens junctions. EMBO Rep. 19, 337-350. doi: 10.15252/embr.20174 4777

El-Gebali, S., Mistry, J., Bateman, A., Eddy, S. R., Luciani, A., Potter, S. C., et al. (2019). The Pfam protein families database in 2019. Nucleic Acids Res. 47, D427-D432. doi: 10.1093/nar/gky995

Emoto, K., He, Y., Ye, B., Grueber, W. B., Adler, P. N., Jan, L. Y., et al. (2004). Control of dendritic branching and tiling by the Tricornered-kinase/Furry signaling pathway in Drosophila sensory neurons. Cell 119, 245-256. doi: 10. 1016/j.cell.2004.09.036

Emoto, K., Jay, Z. P., Jan, L. Y., and Jan, Y.-N. (2006). The tumour suppressor Hippo acts with the NDR kinases in dendritic tiling and maintenance. Nature 443, 210-213. doi: 10.1038/nature05090

Fallahi, E., O'Driscoll, N., and Matallanas, D. (2016). The MST/hippo pathway and cell death: a non-canonical affair. Genes 7:28. doi: 10.3390/genes7060028

Fang, X., and Adler, P. N. (2010). Regulation of cell shape, wing hair initiation and the actin cytoskeleton by Trc/Fry and Wts/Mats complexes. Dev. Biol. 341, 360-374. doi: 10.1016/j.ydbio.2010.02.029

Fang, X., Lu, Q., Emoto, K., and Adler, P. (2010). The Drosophila Fry protein interacts with $\operatorname{Trc}$ and is highly mobile in vivo. BMC Dev. Biol. 10:40. doi: 10.1186/1471-213X-10-40

Favre, B., Turowski, P., and Hemmings, B. A. (1997). Differential inhibition and posttranslational modification of protein phosphatase 1 and 2A in MCF7 cells treated with calyculin-A, okadaic acid, and tautomycin. J. Biol. Chem. 272, 13856-13863. doi: 10.1074/jbc.272.21.13856

Ferrus, A. (1976). Aislamiento y Caracterizacion de Mutantes Morfogeneticos en Drosophila melanogster. Ph. D. Thesis, Universidad Autonoma de Madrid, Madrid, 42.

Fletcher, G. C., Diaz-de-La-Loza, M.-D.-C., Borreguero-Muñoz, N., Holder, M., Aguilar-Aragon, M., and Thompson, B. J. (2018). Mechanical strain regulates the Hippo pathway in. Development 145, dev159467. doi: 10.1242/dev.15 9467

Frenz, L. M., Lee, S. E., Fesquet, D., and Johnston, L. H. (2000). The budding yeast Dbf2 protein kinase localises to the centrosome and moves to the bud neck in late mitosis. J. Cell Sci. 113(Pt 19), 3399-3408.

Fu, C., Iyer, P., Herkal, A., Abdullah, J., Stout, A., and Free, S. J. (2011). Identification and characterization of genes required for cell-to-cell fusion in Neurospora crassa. Eukaryot Cell 10, 1100-1109. doi: 10.1128/EC.05003-11

Fulford, A., Tapon, N., and Ribeiro, P. S. (2018). Upstairs, downstairs: spatial regulation of Hippo signalling. Curr. Opin. Cell Biol. 51, 22-32. doi: 10.1016/ j.ceb.2017.10.006

Fulvia, V., David, J. W., and Paul, N. (1998). Fission yeast orb6, a ser/thr protein kinase related to mammalian rho kinase and myotonic dystrophy kinase, is required for maintenance of cell polarity and coordinates cell morphogenesis with the cell cycle. Proc. Natl. Acad. Sci. U.S.A. 95:7526. doi: 10.1073/pnas.95. 13.7526

Fulvia, V., Juan, M., and Paul, N. (1995). Fission yeast cell morphogenesis: identification of new genes and analysis of their role during the cell cycle. J. Cell Biol. 131, 1529-1538. doi: 10.1083/jcb.131.6.1529

Furth, N., and Aylon, Y. (2017). The LATS1 and LATS2 tumor suppressors: beyond the Hippo pathway. Cell Death Differ. 24, 1488-1501. doi: 10.1038/cdd.2017.99

Gallegos, M. E., and Bargmann, C. I. (2004). Mechanosensory Neurite Termination and Tiling Depend on SAX-2 and the SAX-1 Kinase. Neuron 44, 239-249. doi: 10.1016/j.neuron.2004.09.021

Gates, J. (2012). Drosophila egg chamber elongation: insights into how tissues and organs are shaped. Fly 6, 213-227. doi: 10.4161/fly.21969 
Geng, W., He, B., Wang, M., and Adler, P. (2000). The tricornered gene, which is required for the integrity of epidermal cell extensions, encodes the Drosophila nuclear DBF2-related kinase. Genetics 156, 1817-1828.

Gil-Ranedo, J., Gonzaga, E., Jaworek, K. J., Berger, C., Bossing, T., and Barros, C. S. (2019). STRIPAK members orchestrate hippo and insulin receptor signaling to promote neural stem cell reactivation. Cell Rep. 27, 2921.e5-2933.e5. doi: 10.1016/j.celrep.2019.05.023

Glatter, T., Wepf, A., Aebersold, R., and Gstaiger, M. (2009). An integrated workflow for charting the human interaction proteome: insights into the PP2A system. Mol. Syst. Biol. 5:237. doi: 10.1038/msb.2008.75

Gógl, G., Schneider, K. D., Yeh, B. J., Alam, N., Nguyen Ba, A. N., Moses, A. M., et al. (2015). The structure of an NDR/LATS kinase-mob complex reveals a novel kinase-coactivator system and substrate docking mechanism. Plos Biol. 13:e1002146. doi: 10.1371/journal.pbio.1002146

Gokhale, R., and Pfleger, C. M. (2019). The power of Drosophila Genetics: the discovery of the hippo pathway. Methods Mol. Biol. 1893, 3-26. doi: 10.1007/ 978-1-4939-8910-2_1

Gomez, V., Gundogdu, R., Gomez, M., Hoa, L., Panchal, N., O’Driscoll, M., et al. (2015). Regulation of DNA damage responses and cell cycle progression by hMOB2. Cell Signal. 27, 326-339. doi: 10.1016/j.cellsig.2014.11.016

Gordon, J., Hwang, J., Carrier, K. J., Jones, C. A., Kern, Q. L., Moreno, C. S., et al. (2011). Protein phosphatase 2a (PP2A) binds within the oligomerization domain of striatin and regulates the phosphorylation and activation of the mammalian Ste20-Like kinase Mst3. Bmc Biochem. 12:54. doi: 10.1186/14712091-12-54

Goto, T., Fukui, A., Shibuya, H., Keller, R., and Asashima, M. (2010). Xenopus furry contributes to release of microRNA gene silencing. Proc. Natl. Acad. Sci. U.S.A. 107:19344. doi: 10.1073/pnas.1008954107

Goudreault, M., D’Ambrosio, L. M., Kean, M. J., Mullin, M. J., Larsen, B. G., Sanchez, A., et al. (2009). A PP2A phosphatase high density interaction network identifies a novel striatin-interacting phosphatase and kinase complex linked to the cerebral cavernous malformation 3 (CCM3) protein. Mol. Cell.Proteomics 8, 157-171. doi: 10.1074/mcp.M800266-MCP200

Gruneberg, U., Campbell, K., Simpson, C., Grindlay, J., and Schiebel, E. (2001). Nud1p links astral microtubule organization and the control of exit from mitosis. EMBO J. 20, 305-305. doi: 10.1093/emboj/20.1.305

Gubb, D., and García-Bellido, A. (1982). A genetic analysis of the determination of cuticular polarity during development in Drosophila melanogaster. Development 68, 37-57. doi: 10.1007/BF03349470

Gundogdu, R., and Hergovich, A. (2019). MOB (Mps one Binder) proteins in the hippo pathway and cancer. Cells 8:569. doi: 10.3390/cells8060569

Hachet, O., Bendezú, F. O., and Martin, S. G. (2012). Fission yeast: in shape to divide. Curr. Opin. Cell Biol. 24, 858-864. doi: 10.1016/j.ceb.2012.10.001

Hall, B. G. (2013). Building phylogenetic trees from molecular data with MEGA. Mol. Biol. Evol. 30, 1229-1235. doi: 10.1093/molbev/mst012

Hamaratoglu, F., Maria, W., Madhuri, K.-S., Riitta, N., Eric, H., Chunyao, T., et al. (2005). The tumour-suppressor genes NF2/Merlin and Expanded act through Hippo signalling to regulate cell proliferation and apoptosis. Nat. Cell Biol. 8, 27-36. doi: 10.1038/ncb1339

Hanks, S., and Hunter, T. (1995). Protein kinases 6. The eukaryotic protein kinase superfamily: kinase (catalytic) domain structure and classification. Faseb J. 9, 576-596. doi: 10.1096/fasebj.9.8.7768349

Harvey, K. F., Pfleger, C. M., and Hariharan, I. K. (2003). The Drosophila Mst Ortholog, hippo. restricts growth and cell proliferation and promotes Apoptosis. Cell 114, 457-467. doi: 10.1016/S0092-8674(03)00557-9

He, Y., Emoto, K., Fang, X., Ren, N., Tian, X., Jan, Y.-N., et al. (2005a). ). Drosophila Mob Family Proteins Interact with the Related Tricornered $(\operatorname{Trc})$ and Warts (Wts) Kinases. Mol. Biol. Cell 16, 4139-4152. doi: 10.1091/mbc.e05-01-0018

He, Y., Fang, X., Emoto, K., Jan, Y.-N., and Adler, P. (2005b). The Tricornered Ser/thr protein kinase is regulated by phosphorylation and interacts with furry during Drosophila wing hair development. Mol. Biol. Cell 16, 689-700. doi: 10.1091/mbc.E04-09-0828

Hergovich, A. (2011). MOB control: reviewing a conserved family of kinase regulators. Cell. Signal. 23, 1433-1440. doi: 10.1016/j.cellsig.2011.04.007

Hergovich, A. (2013). Regulation and functions of mammalian LATS/NDR kinases: looking beyond canonical Hippo signalling. Cell Biosci. 3:32. doi: 10.1186/20453701-3-32
Hergovich, A. (2016). The roles of NDR protein kinases in hippo signalling. Genes 7, E21. doi: 10.3390/genes7050021

Hergovich, A., Bichsel, S. J., and Hemmings, B. A. (2005). Human NDR kinases are rapidly activated by $\mathrm{MOB}$ proteins through recruitment to the plasma membrane and phosphorylation. Mol. Cell. Biol. 25, 8259-8272. doi: 10.1128/ MCB.25.18.8259-8272.2005

Hergovich, A., and Hemmings, B. A. (2012). Hippo signalling in the G2/M cell cycle phase: Lessons learned from the yeast MEN and SIN pathways. Semin. Cell Dev. Biol. 23, 794-802. doi: 10.1016/j.semcdb.2012.04.001

Hergovich, A., Schmitz, D., and Hemmings, B. A. (2006a). The human tumour suppressor LATS1 is activated by human MOB1 at the membrane. Biochem. Biophys. Res. Commun. 345, 50-58. doi: 10.1016/j.bbrc.2006.03.244

Hergovich, A., Stegert, M. R., Schmitz, D., and Hemmings, B. A. (2006b). NDR kinases regulate essential cell processes from yeast to humans. Nat. Rev. Mol. Cell Biol. 7:253. doi: 10.1038/nrm1891

Hirata, D., Kishimoto, N., Suda, M., Sogabe, Y., Nakagawa, S., Yoshida, Y., et al. (2002). Fission yeast Mor2/Cps12, a protein similar to Drosophila furry, is essential for cell morphogenesis and its mutation induces Weel-dependent G 2 delay. EMBO J. 21, 4863-4874. doi: 10.1093/emboj/cdf495

Ho, L.-L., Wei, X., Shimizu, T., and Lai, Z.-C. (2010). Mob as tumor suppressor is activated at the cell membrane to control tissue growth and organ size in Drosophila. Dev. Biol. 337, 274-283. doi: 10.1016/j.ydbio.2009.10.042

Horne-Badovinac, S., Hill, J., Gerlach, G., Menegas, W., and Bilder, D. (2012). A screen for round egg mutants in Drosophila identifies tricornered. Furry, and Misshapen as Regulators of Egg Chamber Elongation. G3: Genes| Genomes| Genetics 2, 371-378. doi: 10.1534/g3.111.001677

Hotz, M., and Barral, Y. (2014). The mitotic exit network: new turns on old pathways. Trends Cell Biol. 24, 145-152. doi: 10.1016/j.tcb.2013.09.010

Hou, M. C., Salek, J., and McCollum, D. (2000). Mob1p interacts with the Sid2p kinase and is required for cytokinesis in fission yeast. Curr. Biol. 10, 619-622. doi: 10.1016/S0960-9822(00)00492-9

Hou, M.-C., Wiley, D. J., Verde, F., and McCollum, D. (2003). Mob2p interacts with the protein kinase Orb6p to promote coordination of cell polarity with cell cycle progression. J. Cell Sci. 116:125. doi: 10.1242/jcs.00206

Huang, J., Wu, S., Barrera, J., Matthews, K., and Pan, D. (2005). The hippo signaling pathway coordinately regulates cell proliferation and Apoptosis by Inactivating yorkie, the Drosophila Homolog of YAP. Cell 122, 421-434. doi: 10.1016/j.cell. 2005.06.007

Hwang, J., and Pallas, D. C. (2014). STRIPAK complexes: structure, biological function, and involvement in human diseases. Int. J. Biochem. Cell Biol. 47, 118-148. doi: 10.1016/j.biocel.2013.11.021

Hyodo, T., Ito, S., Hasegawa, H., Asano, E., Maeda, M., Urano, T., et al. (2012). Misshapen-like kinase 1 (MINK1) is a novel component of striatin-interacting phosphatase and kinase (STRIPAK) and is required for the completion of cytokinesis. J. Biol. Chem. 287, 25019-25029. doi: 10.1074/jbc.M112.372342

Ibar, C., Kirichenko, E., Keepers, B., Enners, E., Fleisch, K., and Irvine, K. D. (2018). Tension-dependent regulation of mammalian Hippo signaling through LIMD1. J. Cell Sci. 131, jcs214700. doi: 10.1242/jcs.214700

Irvine, K. D., and Harvey, K. F. (2015). Control of organ growth by patterning and hippo signaling in Drosophila. Cold Spring Harbor Perspect. Biol. 7:a019224. doi: $10.1101 /$ cshperspect.a019224

Jan, Y.-N., and Jan, L. Y. (2010). Branching out: mechanisms of dendritic arborization. Nat. Rev. Neurosci. 11, 316-328. doi: 10.1038/nrn2836

Jia, J., Zhang, W., Wang, B., Trinko, R., and Jiang, J. (2003). The Drosophila Ste20 family kinase dMST functions as a tumor suppressor by restricting cell proliferation and promoting apoptosis. Genes Dev. 17, 2514-2519. doi: 10.1101/ gad. 1134003

Joffre, C., Codogno, P., Fanto, M., Hergovich, A., and Camonis, J. (2016). STK38 at the crossroad between autophagy and apoptosis. Autophagy 12, 594-595. doi: 10.1080/15548627.2015.1135283

Joffre, C., Dupont, N., Hoa, L., Gomez, V., Pardo, R., Gonçalves-Pimentel, C., et al. (2015). The Pro-apoptotic STK38 kinase is a new beclin1 partner positively regulating autophagy. Curr. Biol. 25, 2479-2492. doi: 10.1016/j.cub.2015.08.031

Johnston, L. H., Eberly, S. L., Chapman, J. W., Araki, H., and Sugino, A. (1990). The product of the Saccharomyces cerevisiae cell cycle gene DBF2 has homology with protein kinases and is periodically expressed in the cell cycle. Mol. Cell. Biol. 10:1358. doi: 10.1128/MCB.10.4.1358 
Johnston, L. H., and Thomas, A. P. (1982). The isolation of new DNA synthesis mutants in the yeast Saccharomyces cerevisiae. MGG Mol. Gen. Genet. 186, 439-444. doi: 10.1007/BF00729466

Jones, D. T., Taylor, W. R., and Thornton, J. M. (1992). The rapid generation of mutation data matrices from protein sequences. Comput. Appl. Biosci. 8, 275-282. doi: 10.1093/bioinformatics/8.3.275

Justice, R. W., Zilian, O., Woods, D. F., Noll, M., and Bryant, P. J. (1995). The Drosophila tumor suppressor gene warts encodes a homolog of human myotonic dystrophy kinase and is required for the control of cell shape and proliferation. Genes Dev. 9, 534-546. doi: 10.1101/gad.9.5.534

Kameshita, I., Shimomura, S., Nishio, K., Sueyoshi, N., Nishida, T., Nomura, M., et al. (2010). Expression and characterization of PKL01, an Ndr kinase homolog in. J. Biochem. 147, 799-807. doi: 10.1093/jb/mvq011

Kango-Singh, M., Nolo, R., Tao, C., Verstreken, P., Hiesinger, P. R., Bellen, H. J., et al. (2002). Shar-pei mediates cell proliferation arrest during imaginal disc growth in Drosophila. Development 129, 5719-5730. doi: 10.1242/dev. 00168

Katayama, S., Sugiyama, Y., Hatano, N., Terachi, T., Sueyoshi, N., and Kameshita, I. (2012). PKL01, an Ndr kinase homologue in plant, shows tyrosine kinase activity. J. Biochem. 152, 347-353. doi: 10.1093/jb/mvs075

Kim, S.-Y., Yuka, T., Tomoyuki, M., and Toshio, H. (2016). Structural basis for autoinhibition and its relief of MOB1 in the Hippo pathway. Sci. Rep. 6:e28488. doi: $10.1038 /$ srep 28488

Kim, Y., and Jho, E. H. (2018a). Regulation of the Hippo signaling pathway by ubiquitin modification. BMB Rep. 51, 143-150. doi: 10.5483/BMBRep.2018.51. 3.017

Kim, W., and Jho, E. H. (2018b). The history and regulatory mechanism of the Hippo pathway. BMP Rep. 51, 106-118. doi: 10.5483/bmbrep.2018.51.3.022

Klimek, C., Jahnke, R., Wordehoff, J., Kathage, B., Stadel, D., Behrends, C., et al. (2019). The Hippo network kinase STK38 contributes to protein homeostasis by inhibiting BAG3-mediated autophagy. Biochim. Biophys. Acta Mol. Cell Res. 1866, 1556-1566. doi: 10.1016/j.bbamcr.2019.07.007

Kohler, R. S., Schmitz, D., Cornils, H., Hemmings, B. A., and Hergovich, A. (2010). Differential NDR/LATS interactions with the human MOB family reveal a negative role for human MOB2 in the regulation of human NDR kinases. Mol. Cell. Biol. 30:4507. doi: 10.1128/MCB.00150-10

Koike-Kumagai, M., Yasunaga, K. I., Morikawa, R., Kanamori, T., and Emoto, K. (2009). The target of rapamycin complex 2 controls dendritic tiling of Drosophila sensory neurons through the Tricornered kinase signalling pathway. .EMBO J. 28, 3879-3892. doi: 10.1038/emboj.2009.312

Komarnitsky, S. I., Chiang, Y.-C., Luca, F. C., Chen, J., Toyn, J. H., Winey, M., et al. (1998). DBF2 protein kinase binds to and acts through the cell cycle-regulated MOB1 Protein. Mol. Cell. Biol. 18, 2100-2107. doi: 10.1128/MCB.18.4. 2100

Krapp, A., and Simanis, V. (2008). An overview of the fission yeast septation initiation network (SIN). Biochem. Soc. Trans. 36, 411-415. doi: 10.1042/ BST0360411

Kuck, U., Radchenko, D., and Teichert, I. (2019). STRIPAK, a highly conserved signaling complex, controls multiple eukaryotic cellular and developmental processes and is linked with human diseases. Biol. Chem. doi: 10.1515/hsz2019-0173 [Epub ahead of print].

Kulaberoglu, Y., Lin, K., Holder, M., Gai, Z., Gomez, M., Assefa Shifa, B., et al. (2017). Stable MOB1 interaction with Hippo/MST is not essential for development and tissue growth control. Nat. Commun. 8, 695-695. doi: 10. 1038/s41467-017-00795-y

Kumar, S., Stecher, G., Li, M., Knyaz, C., and Tamura, K. (2018). MEGA X: molecular evolutionary genetics analysis across computing platforms. Mol. Biol. Evol. 35, 1547-1549. doi: 10.1093/molbev/msy096

Kwon, Y., Vinayagam, A., Sun, X., Dephoure, N., Gygi, S. P., Hong, P., et al. (2013). The Hippo signaling pathway interactome.(REPORTS)(Report)(Author abstract). Science 342:737. doi: 10.1126/science.1243971

Lai, Z.-C., Wei, X., Shimizu, T., Ramos, E., Rohrbaugh, M., Nikolaidis, N., et al. (2005). Control of cell proliferation and apoptosis by mob as tumor suppressor. Mats. Cell 120, 675-685. doi: 10.1016/j.cell.2004.12.036

Leger, H., Santana, E., Leu, N. A., Smith, E. T., Beltran, W. A., Aguirre, G. D., et al. (2018). Ndr kinases regulate retinal interneuron proliferation and homeostasis. Sci. Rep. 8:12544. doi: 10.1038/s41598-018-30492-9
Lewellyn, L., Cetera, M., and Horne-Badovinac, S. (2013). Misshapen decreases integrin levels to promote epithelial motility and planar polarity in Drosophila. J. Cell Biol. 200, 721-729. doi: 10.1083/jcb.201209129

Li, Q., Li, S., Mana-Capelli, S., Roth flach, R. J., Danai, L. V., Amcheslavsky, A., et al. (2014). The conserved misshapen-warts-yorkie pathway acts in enteroblasts to regulate intestinal stem cells in Drosophila. Dev. Cell 31, 291-304. doi: 10.1016/ j.devcel.2014.09.012

Li, S., Cho, Y., Yue, T., Ip, Y., and Jiang, J. (2015). Overlapping functions of the MAP4K family kinases Hppy and Msn in Hippo signaling. Cell Discov. 1:15038. doi: 10.1038/celldisc.2015.38

Lignitto, L., Arcella, A., Sepe, M., Rinaldi, L., Delle Donne, R., and Gallo, A. (2013). Proteolysis of MOB1 by the ubiquitin ligase praja2 attenuates Hippo signalling and supports glioblastoma growth. Nat. Commun 2013:1822. doi: $10.1038 /$ ncomms 2791

Ling, P., Lu, T.-J., Yuan, C.-J., and Lai, M.-D. (2008). Biosignaling of mammalian Ste20-related kinases. Cell. Signal. 20, 1237-1247. doi: 10.1016/j.cellsig.2007. 12.019

Liu, L.-Y., Lin, C.-H., and Fan, S.-S. (2009). Function of Drosophila mob2 in photoreceptor morphogenesis. Cell Tissue Res. 338, 377-389. doi: 10.1007/ s00441-009-0878-7

Liu, X., and Winey, M. (2012). The MPS1 family of protein kinases. Annu. Rev. Biochem. 81, 561-585. doi: 10.1146/annurev-biochem-061611-090435

London, N., and Biggins, S. (2014). Signalling dynamics in the spindle checkpoint response. Nat. Rev. Mol. Cell Biol. 15, 735-747. doi: 10.1038/nrm3888

Luca, F. C., and Winey, M. (1998a). Regulation of Moblp, an essential budding yeast protein required for completion of mitosis and spindle pole body duplication. Mol. Biol. Cell 9:12A.

Luca, F. C., and Winey, M. (1998b). MOB1, an essential yeast gene required for completion of mitosis and maintenance of ploidy. Mol. Biol. Cell 9, 29-46. doi: $10.1091 / \mathrm{mbc} .9 .1 .29$

Madsen, C. D., Hooper, S., Tozluoglu, M., Bruckbauer, A., Fletcher, G., Erler, J. T., et al. (2015). STRIPAK components determine mode of cancer cell migration and metastasis. Nat. Cell Biol. 17, 68-80. doi: 10.1038/ncb3083

Maerz, S., Dettmann, A., Ziv, C., Liu, Y., Valerius, O., Yarden, O., et al. (2009). Two NDR kinase-MOB complexes function as distinct modules during septum formation and tip extension in Neurospora crassa. Mol. Microbiol. 74, 707-723. doi: 10.1111/j.1365-2958.2009.06896.x

Maerz, S., and Seiler, S. (2010). Tales of RAM and MOR: NDR kinase signaling in fungal morphogenesis. Curr. Opin. Microbiol. 13, 663-671. doi: 10.1016/j.mib. 2010.08.010

Mah, A. S., Jang, J., and Deshaies, R. J. (2001). Protein kinase Cdc15 activates the Dbf2-Mob1 kinase complex. Proc. Natl.Acad. Scie. U.S.A. 98, 7325-7330. doi: $10.1073 /$ pnas. 141098998

Mana-Capelli, S., and McCollum, D. (2018). Angiomotins stimulate LATS kinase autophosphorylation and act as scaffolds that promote Hippo signaling. J. Biol. Chem. 293, 18230-18241. doi: 10.1074/jbc.RA118.004187

Manning, S. A., and Harvey, K. F. (2015). Warts opens up for activation. Dev. Cell 35, 666-668. doi: 10.1016/j.devcel.2015.12.004

Martin, A. P., Jacquemyn, M., Lipecka, J., Chhuon, C., Aushev, V. N., Meunier, B., et al. (2019). STK38 kinase acts as XPO1 gatekeeper regulating the nuclear export of autophagy proteins and other cargoes. EMBO Rep. .20:e48150. doi: $10.15252 / \mathrm{embr} .201948150$

Matsui, Y., and Lai, Z.-C. (2013). Mutual regulation between Hippo signaling and actin cytoskeleton. Protein Cell 4, 904-910. doi: 10.1007/s13238-013-3084-z

Meignin, C., Alvarez-Garcia, I., Davis, I., and Palacios, I. M. (2007). The salvador-warts-hippo pathway is required for epithelial proliferation and axis specification in Drosophila. Curr. Bio. 17, 1871-1878. doi: 10.1016/j.cub.2007. 09.062

Meng, Z., Moroishi, T., Mottier-Pavie, V., Plouffe, S. W., Hansen, C. G., Hong, A. W., et al. (2015). MAP4K family kinases act in parallel to MST1/2 to activate LATS1/2 in the Hippo pathway. Nat. Commun. 6:8357. doi: 10.1038/ ncomms 9357

Meserve, J. H., and Duronio, R. J. (2015). Scalloped and Yorkie are required for cell cycle re-entry of quiescent cells after tissue damage. Development 142, 2740-2751. doi: 10.1242/dev.119339

Miller, C. J., Lou, H. J., Simpson, C., van de Kooij, B., Ha, B. H., Fisher, O. S., et al. (2019). Comprehensive profiling of the STE20 kinase family defines 
features essential for selective substrate targeting and signaling output. PLoS Bio. 17:e2006540. doi: 10.1371/journal.pbio.2006540

Millward, T., Cron, P., and Hemmings, B. A. (1995). Molecular cloning and characterization of a conserved nuclear serine(threonine) protein kinase. Proc. Natl.l Acad. Scie. U.S.A. 92, 5022-5026. doi: 10.1073/pnas.92.11.5022

Millward, T. A., Hess, D., and Hemmings, B. A. (1999). Ndr protein kinase is regulated by phosphorylation on two conserved sequence motifs. J. Biol. Chem. 274, 33847-33850. doi: 10.1074/jbc.274.48.33847

Misra, J. R., and Irvine, K. D. (2018). The hippo signaling network and its biological functions. Annu. Rev. Genet. 52, 65-87. doi: 10.1146/annurev-genet-120417031621

Mrkobrada, S., Boucher, L., Tyers, D., and Sicheri, F. (2006). Structural and functional analysis of Saccharomyces Cerevisiae Mob1. J. Mol. Biol. 362, 430-440. doi: 10.1016/j.jmb.2006.07.007

Nagai, T., and Mizuno, K. (2014). Multifaceted roles of Furry proteins in invertebrates and vertebrates. J. Biochem. 155, 137-146. doi: 10.1093/jb/ muv001

Nasr, F., Becam, A. M., and Herbert, C. J. (1996). The sequence of $36.8 \mathrm{~kb}$ from the left arm of chromosome XIV reveals 24 complete open reading frames: 18 correspond to new genes, one of which encodes a protein similar to the human myotonic dystrophy kinase. Yeast 12, 169-175. doi: 10.1002/(sici)10970061(199602)12:2<169::aid-yea894>3.0.co;2-b

Natarajan, R., Kara, B., Amanda, B., Phillip, C., Anuoluwapo, E., and Yogesh, P. W. (2015). Tricornered KINASE REGULATES SYNAPSE DEVELOPMENT BY REGULATING THE LEVELS OF WISKOTT-ALDRICH SYNDROME Protein. PLoS One 10:e0138188. doi: 10.1371/journal.pone.0138188

Neisch, A. L., Neufeld, T. P., and Hays, T. S. (2017). A STRIPAK complex mediates axonal transport of autophagosomes and dense core vesicles through PP2A regulation. J. Cell Biol. 216, 441-461. doi: 10.1083/jcb.201606082

Nelson, B., Kurischko, C., Horecka, J., Mody, M., Nair, P., Pratt, L., et al. (2003). RAM: a conserved signaling network that regulates ace $2 p$ transcriptional activity and polarized morphogenesis. Mol. Biol. Cell 14, 3782-3803. doi: 10. 1091/mbc.E03-01-0018

Ni, L., Li, S., Yu, J., Min, J., Brautigam, C. A., Tomchick, D. R., et al. (2013). Structural basis for autoactivation of human mst2 kinase and its regulation by RASSF5. Structure 21, 1757-1768. doi: 10.1016/j.str.2013.07.008

Ni, L., Zheng, Y., Hara, M., Pan, D., and Luo, X. (2015). Structural basis for Mob1dependent activation of the core Mst-Lats kinase cascade in Hippo signaling. Genes Dev. 29, 1416-1431. doi: 10.1101/gad.264929.115

Norkett, R., del Castillo, U., Lu, W., and Gelfand, V. I. (2019). Ser/Thr kinase Trc controls neurite outgrowth in Drosophila by modulating microtubulemicrotubule sliding. bioRxiv doi: 10.7554/eLife.52009 [Preprint].

Oh, H., and Irvine, K. D. (2008). In vivo regulation of Yorkie phosphorylation and localization. Development 135, 1081-1088. doi: 10.1242/dev.015255

Oh, H., and Irvine, K. D. (2009). In vivo analysis of Yorkie phosphorylation sites. Oncogene 28, 1916-1927. doi: 10.1038/onc.2009.43

Oh, H., Reddy, B. V. V. G., and Irvine, K. D. (2009). Phosphorylation-independent repression of Yorkie in Fat-Hippo signaling. Dev. Biol. 335, 188-197. doi: 10. 1016/j.ydbio.2009.08.026

Otsubo, K., Goto, H., Nishio, M., Kawamura, K., Yanagi, S., Nishie, W., et al. (2017). MOB1-YAP1/TAZ-NKX2. 1 axis controls bronchioalveolar cell differentiation, adhesion and tumour formation. Oncogene 36, 4201-4211. doi: 10.1038/onc. 2017.58

Pantalacci, S., Nicolas, T., and Pierre, L. (2003). The Salvador partner Hippo promotes apoptosis and cell-cycle exit in Drosophila. Nat. Cell Biol. 5, 921-927. doi: $10.1038 /$ ncb1051

Parker, B. W., Gógl, G., Bálint, M., Hetenyi, C., Remenyi, A., and Weiss, E. L. (2019). Ndr/Lats kinases bind specific Mob-family coactivators through a conserved and modular interface. bioRxiv doi: 10.1101/242115 [Preprint].

Parrish, J. Z. (2016). "Tiling and mosaic spacing of dendrites," in Dendrites: Development and Disease, eds K. Emoto, R. Wong, E. Huang, and C. Hoogenraad (Tokyo: Springer).

Pearce, L. R., Komander, D., and Alessi, D. R. (2010). The nuts and bolts of AGC protein kinases. Nat. Rev. Mol. Cell Biol. 11, 9-22. doi: 10.1038/nrm2822

Plouffe, S. W., Meng, Z., Lin, K. C., Lin, B., Hong, A. W., Chun, J. V., et al. (2016). Characterization of hippo pathway components by gene inactivation. Mol. Cell 64, 993-1008. doi: 10.1016/j.molcel.2016.10.034
Polesello, C., Huelsmann, S., Brown, N. H., and Tapon, N. (2006). The Drosophila RASSF homolog antagonizes the hippo pathway. Curr. Biol. 16, 2459-2465. doi: $10.1016 /$ j.cub.2006.10.060

Polesello, C., and Tapon, N. (2007). Salvador-warts-hippo signaling Promotes Drosophila posterior follicle cell maturation downstream of notch. Curr. Biol. 17, 1864-1870. doi: 10.1016/j.cub.2007.09.049

Ponchon, L., Dumas, C., Kajava, A. V., Fesquet, D., and Padilla, A. (2004). NMR Solution Structure of Mob1, a Mitotic Exit Network Protein and its Interaction with an NDR Kinase Peptide. J. Mol. Biol. 337, 167-182. doi: 10.1016/j.jmb. 2004.01.010

Poon, C. L. C., Lin, J. I., Zhang, X., and Harvey, K. F. (2011). The Sterile 20-like Kinase Tao-1 Controls tissue growth by regulating the salvador-warts-hippo pathway. Dev. Cell 21, 896-906. doi: 10.1016/j.devcel.2011.09.012

Poon, C. L. C., Liu, W., Song, Y., Gomez, M., Kulaberoglu, Y., Zhang, X., et al. (2018). A hippo-like signaling pathway controls tracheal morphogenesis in Drosophila melanogaster. Dev. Cell 47, 564.e4-565. doi: 10.1016/j.devcel.2018. 09.024

Praskova, M., Xia, F., and Avruch, J. (2008). MOBKL1A/MOBKL1B phosphorylation by MST1 and MST2 inhibits cell proliferation. Curr. Biol. 18, 311-321. doi: 10.1016/j.cub.2008.02.006

Racki, W. J., Becam, A. M., Nasr, F., and Herbert, C. J. (2000). Cbklp, a protein similar to the human myotonic dystrophy kinase, is essential for normal morphogenesis in Saccharomyces cerevisiae. Embo J. 19, 4524-4532. doi: 10. 1093/emboj/19.17.4524

Rademacher, E., and Offringa, R. (2012). Evolutionary adaptations of plant AGC kinases: from light signaling to cell polarity regulation. Front. Plant Sci. 3:250. doi: $10.3389 /$ fpls.2012.00250

Rauskolb, C., Sun, S., Sun, G., Pan, Y., and Irvine, K. D. (2014). Cytoskeletal tension inhibits Hippo signaling through an Ajuba-Warts complex. Cell 158, 143-156. doi: $10.1016 /$ j.cell.2014.05.035

Rawat, S. J., and Chernoff, J. (2015). Regulation of mammalian Ste20 (Mst) kinases. Trends Biochem. Sci. 40, 149-156. doi: 10.1016/j.tibs.2015.01.001

Record, C. J., Chaikuad, A., Rellos, P., Das, S., Pike, A. C. W., Fedorov, O., et al. (2010). Structural comparison of human mammalian Ste20-Like kinases (Human MST Kinases). PLoS One 5:e11905. doi: 10.1371/journal.pone.0011905

Reddy, B. V. V. G., and Irvine, K. D. (2011). Regulation of Drosophila glial cell proliferation by Merlin-Hippo signaling. Development 138, 5201-5212. doi: $10.1242 /$ dev.069385

Ren, F., Zhang, L., and Jiang, J. (2010). Hippo signaling regulates Yorkie nuclear localization and activity through 14-3-3 dependent and independent mechanisms. Dev. Biol. 337, 303-312. doi: 10.1016/j.ydbio.2009.10.046

Ribeiro, P. S., Josué, F., Wepf, A., Wehr, M. C., Rinner, O., Kelly, G., et al. (2010). Combined Functional Genomic and Proteomic Approaches Identify a PP2A Complex as a Negative Regulator of Hippo Signaling. Mol. Cell 39, 521-534. doi: 10.1016/j.molcel.2010.08.002

Richter, A. M., Pfeifer, G. P., and Dammann, R. H. (2009). The RASSF proteins in cancer from epigenetic silencing to functional characterization. BBA Rev. Cancer 1796, 114-128. doi: 10.1016/j.bbcan.2009.03.004

Rock, J. M., Lim, D., Stach, L., Ogrodowicz, R. W., Keck, J. M., Jones, M. H., et al. (2013). Activation of the yeast Hippo pathway by phosphorylation-dependent assembly of signaling complexes. Science $340,871-875$. doi: 10.1126/science. 1235822

Sahu, M. R., and Mondal, A. C. (2019). The emerging role of Hippo signaling in neurodegeneration. J. Neurosci. Res. doi: 10.1002/jnr.24551 [Epub ahead of print].

Sakuma, C., Saito, Y., Umehara, T., Kamimura, K., Maeda, N., Mosca, T. J., et al. (2016). The strip-hippo pathway regulates synaptic terminal formation by modulating actin organization at the Drosophila Neuromuscular Synapses. Cell Rep. 16, 2289-2297. doi: 10.1016/j.celrep.2016.07.066

Salimova, E., Sohrmann, M., Fournier, N., and Simanis, V. (2000). The S. pombe orthologue of the S. cerevisiae MOB1 gene is essential and functions in signalling the onset of septum formation. Journal of Cell Sci. 113, 1695-1704.

Sanchez-Sanz, G., Tywoniuk, B., Matallanas, D., Romano, D., Nguyen, L. K., Kholodenko, B. N., et al. (2016). SARAH domain-mediated MST2-RASSF dimeric interactions. PLoS Comput. Biol. 12:e1005051. doi: 10.1371/journal. pcbi. 1005051 
Sasaki, H., Kawano, O., Endo, K., Suzuki, E., Yukiue, H., Kobayashi, Y., et al. (2007). Human MOB1 expression in non-small-cell lung cancer. Clin. Lung Cancer 8, 273-276. doi: 10.3816/CLC.2007.n.006

Scheel, H., and Hofmann, K. (2003). A novel interaction motif. SARAH, connects three classes of tumor suppressor. Curr. Biol. 13, R899-R900. doi: 10.1016/j.cub. 2003.11.007

Schulte, J., Katharine, J. S., Ramon, A. J., Chaohong, W., Yun, S., Pengyu, H., et al. (2010). DMob4/Phocein regulates synapse formation, axonal transport, and microtubule organization. J. Neurosci.30, 5189. doi: 10.1523/JNEUROSCI. 5823-09.2010

Sharif, A. A. D., and Hergovich, A. (2018). The NDR/LATS protein kinases in immunology and cancer biology. Semin. Cancer Biol. 48, 104-114. doi: 10.1016/ j.semcancer.2017.04.010

Shi, Z., Jiao, S., and Zhou, Z. (2016). STRIPAK complexes in cell signaling and cancer. Oncogene 35, 4549-4557. doi: 10.1038/onc.2016.9

Shomin-Levi, H., and Yarden, O. (2017). The neurospora crassa PP2A regulatory subunits RGB1 and B56 are required for proper growth and development and interact with the NDR Kinase COT1. Front. Microbiol. 8:1694. doi: 10.3389/ fmicb.2017.01694

Snigdha, K., Gangwani, K. S., Lapalikar, G. V., Singh, A., and Kango-Singh, M. (2019). Hippo signaling in cancer: lessons from Drosophila Models. Front. Cell Dev. Biol. 7:85. doi: 10.3389/fcell.2019.00085

Soares, H., Carmona, B., Nolasco, S., and Viseu Melo, L. (2019). Polarity in ciliate models: from cilia to cell architecture. Front. Cell Dev. Biol. 7:240. doi: 10.3389/ fcell.2019.00240

Song, Y., Eng, M., and Ghabrial, A. S. (2013). Focal defects in single-celled tubes mutant for cerebral cavernous malformation 3, GCKIII, or NSF2. Dev. Cell 25, 507-519. doi: 10.1016/j.devcel.2013.05.002

Staley, B. K., and Irvine, K. D. (2012). Hippo signaling in Drosophila: recent advances and insights. Dev. Dyn. 241, 3-15. doi: 10.1002/dvdy.22723

Stavridi, E. S., Harris, K. G., Huyen, Y., Bothos, J., Verwoerd, P.-M., Stayrook, S. E., et al. (2003). Crystal structure of a human mobl protein: toward understanding mob-regulated cell cycle pathways. Structure 11, 1163-1170. doi: 10.1016/ S0969-2126(03)00182-5

Stegert, M. R., Hergovich, A., Tamaskovic, R., Bichsel, S. J., and Hemmings, B. A. (2005). Regulation of NDR protein kinase by hydrophobic motif phosphorylation mediated by the mammalian Ste20-like kinase MST3. Mol. Cell. Biol. 25, 11019-11029. doi: 10.1128/mcb.25.24.11019-11029.2005

Su, T., Ludwig, M. Z., Xu, J., and Fehon, R. G. (2017). Kibra and merlin activate the hippo pathway spatially distinct from and independent of expanded. Dev. Cell 40, 478-490. doi: 10.1016/j.devcel.2017.02.004

Sugden, P. H., McGuffin, L. J., and Clerk, A. (2013). SOcK, MiSTs, MASK and STicKs: the GCKIII (germinal centre kinase III) kinases and their heterologous protein-protein interactions. Bioch. J. 454, 13-30. doi: 10.1042/Bj20130219

Sun, S., Reddy, B. V. V. G., and Irvine, K. D. (2015). Localization of Hippo signalling complexes and Warts activation in vivo. Nat.Commun. 6:e8402. doi: $10.1038 /$ ncomms 9402

Takai, A., Sasaki, K., Nagai, H., Mieskes, G., Isobe, M., Isono, K., et al. (1995). Inhibition of specific binding of okadaic acid to protein phosphatase $2 \mathrm{~A}$ by microcystin-LR, calyculin-A and tautomycin: method of analysis of interactions of tight-binding ligands with target protein. Biochem.J. 306(Pt 3), 657-665. doi: 10.1042/bj3060657

Tang, F., Zhang, L., Xue, G., Hynx, D., Wang, Y., Cron, P. D., et al. (2014). hMOB3 modulates MST1 apoptotic signaling and supports tumor growth in glioblastoma multiforme. Cancer Res. 74, 3779-3789. doi: 10.1158/0008-5472. CAN-13-3430

Tang, Y., Chen, M., Zhou, L., Ma, J., Li, Y., Zhang, H., et al. (2019). Architecture, substructures, and dynamic assembly of STRIPAK complexes in Hippo signaling. Cell Discov. 5, 3-3. doi: 10.1038/s41421-018-0077-3

Tapon, N., Harvey, K. F., Bell, D. W., Wahrer, D. C. R., Schiripo, T. A., Haber, D. A., et al. (2002). salvador promotes both cell cycle exit and Apoptosis in Drosophila and is mutated in human cancer cell lines. Cell 110, 467-478. doi: 10.1016/S0092-8674(02)00824-3

Tewari, R., Bailes, E., Bunting, K. A., and Coates, J. C. (2010). Armadillo-repeat protein functions: questions for little creatures. Trends Cell Biol. 20, 470-481. doi: $10.1016 /$ j.tcb.2010.05.003

The UniProt Consortium (2019). UniProt: a worldwide hub of protein knowledge. Nucleic Acids Res. 47, D506-D515. doi: 10.1093/nar/gky1049
Thompson, B., and Sahai, E. (2015). MST kinases in development and disease. J. Cell Biol. 210, 871-882. doi: 10.1083/jcb.201507005

Thurmond, J., Goodman, J. L., Strelets, V. B., Attrill, H., Gramates, L. S., Marygold, S. J., et al. (2019). FlyBase 2.0: the next generation. Nucleic Acids Res. 47, D759-D765. doi: 10.1093/nar/gky1003

Tomlin, G. C., Morrell, J. L., and Gould, K. L. (2002). The spindle pole body protein Cdc11p links Sid4p to the fission yeast septation initiation network. Mol. Biol. Cell 13, 1203-1214. doi: 10.1091/mbc.01-09-0455

Toyn, J. H., Hiroyuki, A., Akio, S., and Johnston, L. H. (1991). The cell-cycleregulated budding yeast gene $\mathrm{DBF} 2$, encoding a putative protein kinase, has a homologue that is not under cell-cycle control. Gene 104, 63-70. doi: 10.1016/ 0378-1119(91)90465-N

Trammell, M. A., Mahoney, N. M., Agard, D. A., and Vale, R. D. (2008). Mob4 plays a role in spindle focusing in Drosophila S2 cells. J. Cell Sci. 121, 1284-1292. doi: $10.1242 /$ jcs. 017210

Udan, R., Kango-Singh, M., Nolo, R., Tao, C., and Halder, G. (2003). Hippo promotes proliferation arrest and apoptosis in the Salvador/Warts pathway. Nat. Cell Biol. 5, 914-920. doi: 10.1038/ncb1050

Ultanir, S. K., Hertz, N. T., Li, G., Ge, W. P., Burlingame, A. L., Pleasure, S. J., et al. (2012). Chemical genetic identification of NDR1/2 kinase substrates AAK1 and Rabin8 Uncovers their roles in dendrite arborization and spine development. Neuron 73, 1127-1142. doi: 10.1016/j.neuron.2012.01.019

Vinson, C. R., and Adler, P. N. (1987). Directional non-cell autonomy and the transmission of polarity information by the frizzled gene of Drosophila. Nature 329, 549-551. doi: 10.1038/329549a0

Vitulo, N., Vezzi, A., Galla, G., Citterio, S., Marino, G., Ruperti, B., et al. (2007). Characterization and evolution of the cell cycle-associated mob domaincontaining proteins in eukaryotes. Evol. Bioinform Online 3, 121-158. doi: $10.1177 / 117693430700300007$

Vrabioiu, A. M., and Struhl, G. (2015). Fat/dachsous signaling Promotes Drosophila wing growth by regulating the conformational state of the NDR kinase warts. Dev. Cell 35, 737-749. doi: 10.1016/j.devcel.2015.11.027

Wei, X., Shimizu, T., and Lai, Z. C. (2007). Mob as tumor suppressor is activated by Hippo kinase for growth inhibition in Drosophila. EMBO J. 26, 1772-1781. doi: 10.1038/sj.emboj.7601630

Weiss, E. L., Kurischko, C., Zhang, C., Shokat, K., Drubin, D. G., and Luca, F. C. (2002). The Saccharomyces cerevisiae Mob2p-Cbk1p kinase complex promotes polarized growth and acts with the mitotic exit network to facilitate daughter cell-specific localization of Ace2p transcription factor. J. Cell Biol. 158, 885-900. doi: $10.1083 /$ jcb.200203094

Wu, S., Huang, J., Dong, J., and Pan, D. (2003). hippo Encodes a Ste-20 family protein kinase that restricts cell proliferation and promotes apoptosis in conjunction with salvador and warts. Cell 114, 445-456. doi: 10.1016/S00928674(03)00549-X

Xiong, J., Cui, X., Yuan, X., Yu, X., Sun, J., and Gong, Q. (2016). The Hippo/STE20 homolog SIK1 interacts with MOB1 to regulate cell proliferation and cell expansion in Arabidopsis. J. Exp. Bot. 67, 1461-1475. doi: 10.1093/jxb/erv538

Xiong, S., Couzens, A., Kean, M., Mao, D., Guettler, S., and Sicheri, F. (2017). Regulation of Protein Interactions by Mps One Binder (MOB1) Phosphorylation. Mol. Cell. Proteomics 16, 1111-1125. doi: 10.1074/mcp.M117. 068130

Xiong, S., Lorenzen, K., Couzens, A. L., Templeton, C. M., Rajendran, D., Mao, D. Y. L., et al. (2018). Structural basis for auto-inhibition of the NDR1 kinase domain by an atypically long activation segment. Structure 26, 1101.e6-1115.e6. doi: 10.1016/j.str.2018.05.014

Xu, T., Wang, W., Zhang, S., Stewart, R. A., and Yu, W. (1995). Identifying tumor suppressors in genetic mosaics: the Drosophila lats gene encodes a putative protein kinase. Development 121, 1053-1063. doi: 10.1002/cyto.990190202

Xu, J., Vanderzalm, P. J., Ludwig, M., Su, T., Tokamov, S. A., and Fehon, R. G. (2018). Yorkie functions at the cell cortex to promote myosin activation in a non-transcriptional manner. Dev. Cell 46, 271.e5-284.e5. doi: 10.1016/j.devcel. 2018.06.017

Yabuta, N., Mukai, S., Okamoto, A., Okuzaki, D., Suzuki, H., Torigata, K., et al. (2013). N-terminal truncation of Lats 1 causes abnormal cell growth control and chromosomal instability. J. Cell Sci. 126, 508. doi: 10.1242/jcs.113431

Yan, M., Chu, L., Qin, B., Wang, Z., Liu, X., Jin, C., et al. (2015). Regulation of NDR1 activity by PLK1 ensures proper spindle orientation in mitosis. Sci. Rep. 5, 10449. doi: 10.1038/srep10449 
Yan, Y., Denef, N., Tang, C., and Schupbach, T. (2011). Drosophila PI4KIIIalpha is required in follicle cells for oocyte polarization and Hippo signaling. Development 138, 1697-1703. doi: 10.1242/dev.059279

Yarden, O., Plamann, M., Ebbole, D. J., and Yanofsky, C. (1992). cot-1, a gene required for hyphal elongation in Neurospora crassa, encodes a protein kinase. EMBO J. 11, 2159-2566. doi: 10.1002/j.1460-2075.1992.tb05275.x

Ye, X., Nikolaidis, N., Nei, M., and Lai, Z.-C. (2009). Evolution of the mob Gene Family. Open Cell Signal J. 1, 1-11. doi: 10.2174/187639010090101 0001

Yin, F., Yu, J., Zheng, Y., Chen, Q., Zhang, N., and Pan, D. (2013). Spatial organization of hippo signaling at the plasma membrane mediated by the tumor suppressor Merlin/NF2. Cell 154, 1342-1355. doi: 10.1016/j.cell.2013.08.025

Yu, F., and Guan, K. (2013). The Hippo pathway: regulators and regulations. Genes Dev. 27, 355-371. doi: 10.1101/gad.210773.112

Yu, J., Poulton, J., Huang, Y.-C., and Deng, W.-M. (2008). The hippo pathway promotes notch signaling in regulation of cell differentiation, proliferation, and oocyte polarity (Hippo Regulates Notch Activity). PLoS One 3:e1761. doi: 10.1371/journal.pone.0001761

Yu, J., Zheng, Y., Dong, J., Klusza, S., Deng, W.-M., and Pan, D. (2010). Kibra functions as a tumor suppressor protein that regulates hippo signaling in conjunction with merlin and expanded. Dev. Cell 18, 288-299. doi: 10.1016/j. devcel.2009.12.012

Yu, T., Bachman, J., and Lai, Z.-C. (2015). Mutation analysis of large tumor suppressor genes LATS1 and LATS2 supports a tumor suppressor role in human cancer. Protein Cell 6, 6-11. doi: 10.1007/s13238-014-0122-4

Yue, T., Tian, A., and Jiang, J. (2012). The Cell adhesion molecule echinoid functions as a tumor suppressor and upstream regulator of the hippo signaling pathway. Dev. Cell 22, 255-267. doi: 10.1016/j.devcel.2011.12.011

Zermiani, M., Begheldo, M., Nonis, A., Palme, K., Mizzi, L., Morandini, P., et al. (2015). Identification of the arabidopsis RAM/MOR signalling network: adding new regulatory players in plant stem cell maintenance and cell polarization. Ann. Bot. 116, 69-89. doi: 10.1093/aob/mcv066

Zhang, L., Ren, F., Zhang, Q., Chen, Y., Wang, B., and Jiang, J. (2008). The TEAD/TEF family of transcription factor scalloped mediates hippo signaling in organ size control. Dev. Cell 14, 377-387. doi: 10.1016/j.devcel.2008.01.006

Zhang, W., Shen, J., Gu, F., Zhang, Y., Wu, W., Weng, J., et al. (2018). Monopolar spindle-one-binder protein 2 regulates the activity of large tumor suppressor/yes-associated protein to inhibit the motility of SMMC-7721 hepatocellular carcinoma cells. Oncol. Lett. 15, 5375-5383. doi: 10.3892/ol.2018. 7952

Zheng, Y., Liu, B., Wang, L., Lei, H., Pulgar Prieto, K. D., and Pan, D. (2017). Homeostatic control of hpo/mst kinase activity through autophosphorylationdependent recruitment of the STRIPAK PP2A phosphatase complex. Cell Rep. 21, 3612-3623. doi: 10.1016/j.celrep.2017.11.076

Zheng, Y., and Pan, D. (2019). The hippo signaling pathway in development and disease. Dev. Cell 50, 264-282. doi: 10.1016/j.devcel.2019.06.003

Zheng, Y., Wang, W., Liu, B., Deng, H., Uster, E., and Pan, D. (2015). Identification of happyhour/MAP4K as alternative Hpo/Mst-like kinases in the hippo kinase cascade. Dev. Cell 34, 642-655. doi: 10.1016/j.devcel.2015.08.014

Conflict of Interest: The authors declare that the research was conducted in the absence of any commercial or financial relationships that could be construed as a potential conflict of interest.

Copyright $\odot 2020$ Duhart and Raftery. This is an open-access article distributed under the terms of the Creative Commons Attribution License (CC BY). The use, distribution or reproduction in other forums is permitted, provided the original author(s) and the copyright owner(s) are credited and that the original publication in this journal is cited, in accordance with accepted academic practice. No use, distribution or reproduction is permitted which does not comply with these terms. 